Review

\title{
Food Insecurity and Major Diet-Related Morbidities in Migrating Children: A Systematic Review
}

\author{
Arianna Dondi ${ }^{1, *} \mathbb{C}$, Valentina Piccinno ${ }^{2}$, Francesca Morigi ${ }^{1}$, Sugitha Sureshkumar ${ }^{3}$, \\ Davide Gori ${ }^{4}$ and Marcello Lanari ${ }^{1}$ \\ 1 Pediatric Emergency Unit, Department of Medical and Surgical Sciences (DIMEC), S.Orsola Hospital, \\ University of Bologna, 40138 Bologna, Italy; francesca.morigi@gmail.com (F.M.); \\ marcello.lanari@unibo.it (M.L.) \\ 2 Pediatric and Neonatology Unit, Imola Hospital, 40026 Imola (Bologna), Italy; valentinapiccinno@gmail.com \\ 3 Institute of Global Health, University of Geneva, 1205 Geneva, Switzerland; sugi84@hotmail.com \\ 4 Department of Biomedical and Neuromotor Sciences, University of Bologna, 40100 Bologna, Italy; \\ davide.gori4@unibo.it \\ * Correspondence: arianna.dondi@gmail.com; Tel.: +3905-1214-3012
}

Received: 30 December 2019; Accepted: 27 January 2020; Published: 31 January 2020

\begin{abstract}
Children of migrant families are known to be at a higher risk of diet-related morbidities due to complex variables including food insecurity, cultural and religious beliefs, and sociodemographic factors like ethnicity, socioeconomic status, and education. Several studies have assessed the presence of specific diseases related to dietary issues in migrant children. This systematic review aims to highlight the existing body of work on nutritional deficiencies in the specific vulnerable pediatric population of immigrants. Refugees were intentionally excluded because of fundamental differences between the two groups including the reasons for migration and health status at the time of arrival. A total of 29 papers were included and assessed for quality. Most of them described a strong correlation between obesity and migration. A high prevalence of stunting, early childhood caries, iron and vitamin D deficiency was also reported, but the studies were few and heterogeneous. Food insecurity and acculturation were found important social factors (nevertheless with inconclusive results) influencing dietary habits and contributing to the development of morbidities such as obesity and other metabolic disorders, which can cause progressive unsustainability of health systems. Public health screening for diet-related diseases in migrant children may be implemented. Educational programs to improve children's diet and promote healthy-living behaviors as a form of socioeconomic investment for the health of the new generations may also be considered.
\end{abstract}

Keywords: migration; children; vulnerable groups; food insecurity; obesity; stunting; vitamin D; iron; early childhood caries; diet

\section{Introduction}

During the course of history migratory patterns have been defined by demands for work, education, escape from armed conflict and poverty, or climate change [1,2]. In the recent past, high-income countries (HIC) have consistently received international migrants with steady increases over time. The estimated number of migrants to Europe and Northern America exceeded the number of emigrants by 25.9 million in the decade 2010-2020 [1,2]. This paper does not make reference to the political aspects of migration surrounding voluntary and involuntary migration (the forced displacement precluding asylum seekers and refugees), and aims to objectively provide the status of nutrition amongst children in migrant populations globally.

Food insecurity (FI) is a common problem among migrant people and entails several issues such as cultural and religious beliefs, socio-demographic, economic, and environmental factors, education, 
and lifestyle changes [3,4]. It has been associated with a number of negative health and behavioral outcomes, mostly pertaining to children [5-8]. The most commonly referenced resource for the definition of Food Security is the US Department of Agriculture, that describes it as "access by all people at all times to enough food for an active, healthy life" [9]. Thus, the inverse proves true for FI.

Several studies point out that immigrant families, compared to non-immigrant ones, disproportionately experience FI, which is a concerning factor given the increasing size of this population [10,11]. Most of the studies about the relationship between FI and migration were conducted in the USA, but the growth rate of FI among children is also a well-known issue in European countries. Up to $18 \%$ of European households experience moderate-to-severe inabilities to access food and up to $20 \%$ report not enough money to buy food $[12,13]$. One of the causes that can be found in the loss of access to types of food that was previously familiar and known, with which they have a cultural and traditional connection. This factor, combined with a low socio-economic setting and a lack of knowledge about the nutritional values of food available in the host country, might contribute to the increase in FI in these populations. All these behaviors have been related to a number of negative health implications, particularly for children and adolescents that are the most vulnerable sub-population, with regards to the age-specific needs [4]. The lack of access to specific nutrients and to a varied and balanced diet, which are essential components for developing organisms, can lead to micronutrient deprivation and predispose to diseases such as anemia and rickets. Iron deficiency (ID) and low levels of vitamin D are common in migrant children in HIC [14,15]. Risk factors for vitamin D deficiency include being of female gender and having dark skin pigmentation. Inadequate levels of vitamin $\mathrm{D}$ prevent proper bone development, which can result in rickets in growing children and osteomalacia when growth has ended [15], while depletion of the iron stores can lead to anemia and, when it starts from early infancy and persists over time, even to neuro-cognitive delay [16].

Migration from low to high resource settings could also lead to FI without hunger, but with malnutrition. Indeed, economic and cultural factors could also lead to dietary simplifications with overconsumption of high energy, low-cost, nutrient-poor, sugary and fatty products and beverages (junk foods), which are directly linked to obesity, metabolic disorders, cardiovascular diseases [17], early childhood caries (ECC) [18,19]. The American Academy of Pediatric Dentistry defines ECC as "the presence of 1 or more decayed (non-cavitated or cavitated lesions), missing (due to caries), or filled tooth surfaces" in any primary tooth in a child 71 months of age or younger [20]. High simple-sugar diet, insufficient exposure to fluoride, poor oral hygiene and lack of financial resources to access dental care are implicated in the development of ECC, which eventually may lead to pain, abscesses, impaired speech, and eating problems [21].

This systematic review aims to highlight the existing body of work on nutritional deficiencies in the specific vulnerable pediatric population of immigrants with the exclusion of refugees. In fact, despite a frequent overlapping of the two terms, there are substantial differences between the two groups: immigrants normally choose to move from their original setting to a new one, whereas refugees are normally forced to move due to geopolitical reasons and are considered to require protection [22]; refugees commonly arrive in worse conditions and are at a higher risk of transitioning to poorer health [23-25]. For these reasons, refugees are seen here as a significantly different population and the study has been limited to immigrants. The research questions addressed include: (1) Is there documented evidence of a correlation between migration, FI, and morbidity amongst the pediatric population? (2) Are there any specified diseases with a clear association to dietary problems and food insecurity in migrant children?

\section{Materials and Methods}

This systematic literature review was performed in November 2019 by browsing into the following databases: Medline (Pubmed), Cochrane Library, Clinicaltrials.gov. Research was improved by searching in the most important websites of guidelines and clearinghouses and in the most important etextbooks sites. 
The Preferred Reporting Items for Systematic Reviews and Meta-analyses guidelines (PRISMA) flow chart [26] was used to guide the transparent exclusion of published literature with defined reasons. The pertinent literature which was selected was hence tabulated with regards to country of origin, migration to, study design, number, age, and migrant characteristics, outcome disease, dietary issues, and main results. Further reporting surrounded bias, data reliability, and quality, to help the decision-making process during designing, implementing, and evaluating health intervention projects and policy.

The present work was registered to the international prospective register of systematic reviews, PROSPERO, with the following registration number: CRD42019145319.

Inclusion Criteria: Date: published between January 2005 and November 2019; Exposure: Migrant children and nutritional status; Population: migrants from low to high resource settings (different regions in the same country to be included); Language: English; Study Design: RCT, cohort studies, cross-sectional, retrospective; Outcomes: reported.

Exclusion Criteria: Exposure: not pertaining to migrant children and nutritional status; Population: refugees; Language: other than English; Study Design: Descriptive studies, reports, protocols; Outcomes: unreported.

Search String: ((migrant* OR transient* OR emigrant* OR immigra* OR refugee* OR migrant[MH]) AND (child OR children) AND (religion OR religio* OR Religious belie* OR religious eth* OR religious pract* OR cultural belie* OR socioeconomic factors OR education OR income OR feeding behaviour OR ethnicity) AND (food insecurity OR food security OR food policy OR food supply OR food insec* OR food sec* OR food poli* OR food suppl* OR processed food OR junk food OR western-style diet OR sugar sweetened beverages OR SSB OR soft drinks OR snack OR snacks OR fast food) AND (metabolic syndr* OR metabolic syndrome OR obes* OR obesity OR hypertension OR cardiovascular morbidity OR CV Morbidity OR malnutrition OR failure to thrive OR dental caries OR rickets OR iron deficiency OR iron defic* OR vitamin defic*)).

Quality Assessment: The Effective Public Health Practice Project (EPHPP) Quality assessment tool [27] for quantitative studies was used to quality assess papers in full text.

Key Definitions: Child: A child is a person 19 years or younger unless national law defines a person to be an adult at an earlier age [28].

Nutritional Status: A requirement of health of a person determined by the diet, levels of nutrients contained in the body and normal metabolic integrity. Normal nutritional status is managed by balance food consumption and normal utilization of nutrients [29].

Nutritional Deficiencies: Nutritional deficiencies, excesses, and imbalances all predispose the cell to injury. Various dietary deficiencies or imbalances of essential amino acids, fatty acids, vitamins, or minerals can lead to muscle wasting, decreased stature, increased susceptibility to infection, metabolic disturbances, and a host of other diseases, depending on which elements are missing from or disproportionate in the diet [30].

Food security: Food security exists when all people, at all times, have physical and economic access to sufficient safe and nutritious food that meets their dietary needs and food preferences for an active and healthy life. The four dimensions being: availability, access, utilization, and stability [31]. The opposite proves true for FI.

Socioeconomic status (SES): is usually measured by determining education, income, occupation, or a composite of these dimensions [32].

Immigrant: From the perspective of the country of arrival, a person who moves into a country other than that of his or her nationality or usual residence, so that the country of destination effectively becomes his or her new country of usual residence [33].

Refugee: a foreign-born individual deemed as requiring protection according to the 1951 Status of Refugee Convention who has been accepted into a host country [22]. Geopolitical events, which include war, conflicts, and climate disasters leading to displaced peoples, also encourage migrations [33]. 
Global migration: The drivers of migration can be conceptualized into 5 categories: economic, social, political, demographic, and environmental factors, the former 2 perceived as having the greatest effect on the volume and patterns of migration [2]. Economic motives move migration both to developed countries and to developing countries with monetary interest. Migrants seeking work but opting to remain within their regions of origin, cross to adjacent countries to satisfy their economic interests. As well as these so-called push factors, migrants are pulled for recruited labor to satiate voids in the low and high-skilled workforce [34-38].

Migrant flow (international): The number of international migrants arriving in a country (immigrants) or the number of international migrants departing from a country (emigrants) over the course of a specific period [33].

\section{Results}

\subsection{Search Yields, Risk of Bias, and Quality of Reporting}

The initial search identified 299 studies (Figure 1). 29 studies were included in the final analysis after 270 papers were excluded on the basis of the inclusion/exclusion criteria. A detailed description of the selected articles, with study characteristics, is reported in Table 7. The quality assessment of the full text papers included in the present systematic review is reported in Table 8.

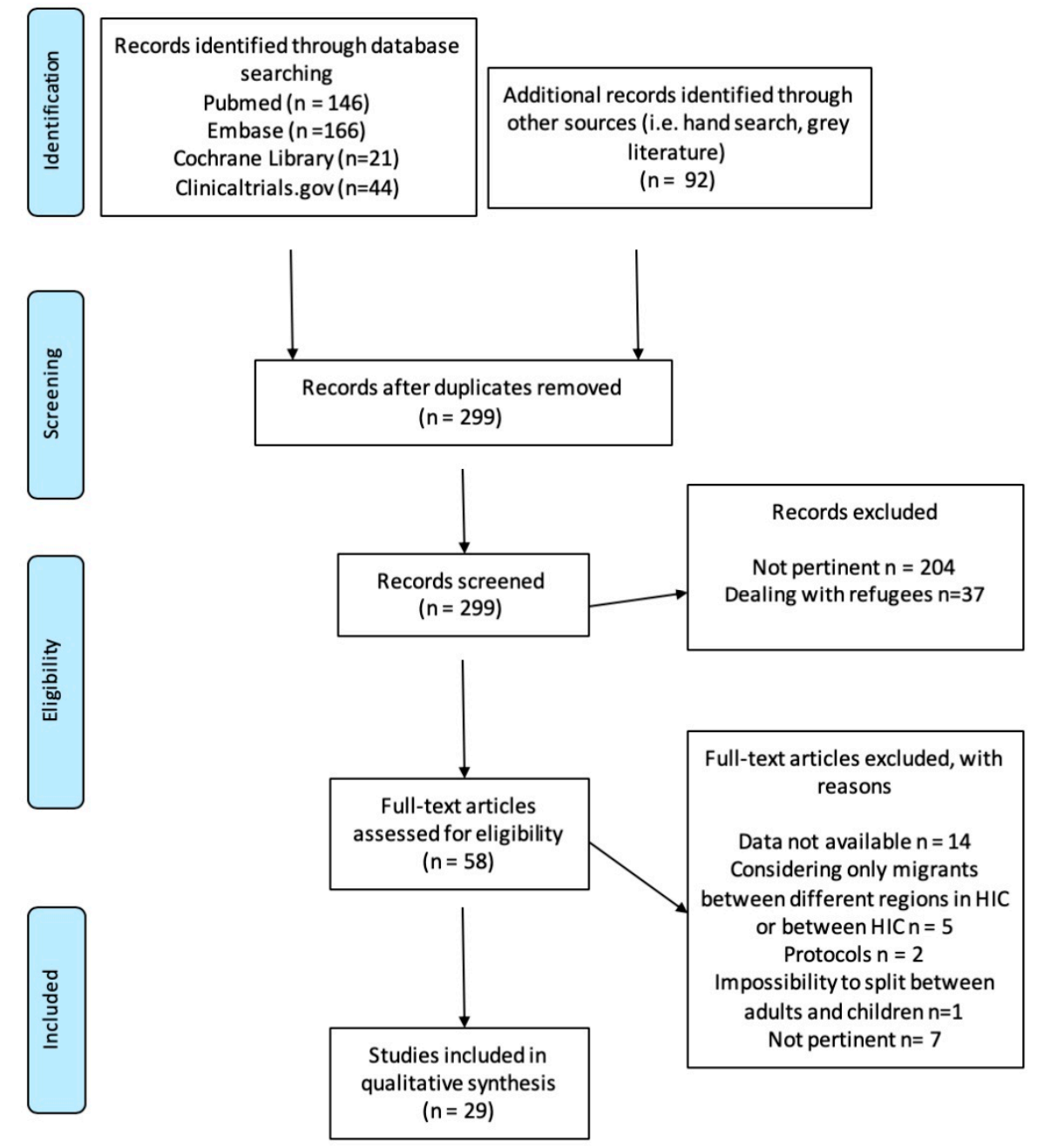

Figure 1. Preferred reporting items for systematic reviews and meta-analyses (PRISMA) 2009 Flow Diagram showing the process for articles selection [26]. HIC= high income countries. 
Table 1. Characteristics and main results of the included studies. [27].

\begin{tabular}{|c|c|c|c|c|c|c|c|c|c|}
\hline Author, Year & $\begin{array}{l}\text { Migrating } \\
\text { from }\end{array}$ & $\begin{array}{c}\text { Immigrating } \\
\text { to }\end{array}$ & Study Design & $\begin{array}{c}\text { N, Migrant } \\
\text { Characteristics }\end{array}$ & Age & Outcome Disease & Dietary/Cultural Issues & Main Results & QA \\
\hline $\begin{array}{c}\text { Rosas, } 2010 \\
{[35]}\end{array}$ & Mexico & $\begin{array}{l}\text { USA } \\
\text { (California) }\end{array}$ & cross-sectional & $\begin{array}{l}603 \text { mother-child pairs } \\
\text { Mexico-born and } \\
\text { California } \\
\text { born-children of } \\
\text { Mexican descent }\end{array}$ & 5 year & overweight/obesity & $\begin{array}{l}\text { Maternal obesity was a } \\
\text { determinant of childhood } \\
\text { obesity in both settings; in } \\
\text { Mexico, male gender, high } \\
\text { SES and low food security } \\
\text { were determinants of } \\
\text { childhood obesity. }\end{array}$ & $\begin{array}{l}\text { prevalence of childhood } \\
\text { obesity is much higher } \\
\text { among children of Mexican } \\
\text { descent }\end{array}$ & 13 \\
\hline $\begin{array}{c}\text { Kaiser, } 2015 \\
{[36]}\end{array}$ & Mexico & $\begin{array}{l}\text { USA (rural } \\
\text { communities } \\
\text { in California) }\end{array}$ & cohort & $\begin{array}{c}217 \text { children of Latino } \\
\text { descent }\end{array}$ & $2-8$ year & overweight/obesity & $\begin{array}{l}\text { Preference of american-style } \\
\text { foods after children enter the } \\
\text { public school system }\end{array}$ & $\begin{array}{l}51 \% \text { of children } \\
\text { overweight/obese }\end{array}$ & 14 \\
\hline $\begin{array}{c}\text { Kobel, } 2017 \\
\quad[37]\end{array}$ & $\begin{array}{l}\text { anywhere } \\
\text { (mostly } \\
\text { Turkey and } \\
\text { Russia) }\end{array}$ & Germany & $\begin{array}{l}\text { randomized } \\
\text { controlled trial }\end{array}$ & $\begin{array}{l}525 \text { children with } \\
\text { migration background }\end{array}$ & $7.1 \pm 0.7$ year & obesity & $\begin{array}{l}\text { Lower fruit and vegetable } \\
\text { consumption, higher screen } \\
\text { media time, lower physical } \\
\text { activity }\end{array}$ & $\begin{array}{l}\text { children with migrant } \\
\text { background: } 11.8 \% \\
\text { overweight/obese (vs } 9 \% \text { non } \\
\text { migrant) }-5.7 \% \text { obesity if } \\
\text { migration background }\end{array}$ & 14 \\
\hline Early 2019 [39] & $\begin{array}{c}\text { Hispanic } \\
(85 \%), \\
\text { Black/African } \\
(3 \%) \\
\end{array}$ & $\begin{array}{c}\text { USA } \\
\text { (California) }\end{array}$ & $\begin{array}{l}\text { quasi-randomized } \\
\text { controlled trial }\end{array}$ & 68 children & 10.8 year & overweight/obesity & $\begin{array}{c}\text { inadequate fruit and } \\
\text { vegetable consumption, } \\
\text { excess of sugar-sweetened } \\
\text { beverages }\end{array}$ & $\begin{array}{c}\text { overweight prevalence: } \\
11.8 \% \text {; obesity prevalence: } \\
54.4 \%\end{array}$ & 12 \\
\hline $\begin{array}{c}\text { Geremia, } 2015 \\
{[40]}\end{array}$ & Italy & Brazil & cross-sectional & $\begin{array}{l}590 \text { Italian immigrant } \\
\text { children }\end{array}$ & 9-18 year & overweight/obesity & $\begin{array}{l}\text { Low frequency of } \\
\text { consumption of vegetables, } \\
\text { more fat foods. }\end{array}$ & $\begin{array}{l}\text { 1) High prevalence of } \\
\text { overweight and obesity in } \\
\text { this city; } 2 \text { ) factors such as } \\
\text { omission of breakfast, } \\
\text { overweight and obesity in } \\
\text { the mother, age and male } \\
\text { gender were associated with } \\
\text { excess weight. }\end{array}$ & 12 \\
\hline $\begin{array}{l}\text { Lane, } 2018 \\
\quad \text { [22] }\end{array}$ & $\begin{array}{l}\text { Asia, Middle } \\
\text { East, Africa, } \\
\text { Latin America, } \\
\text { Europe o US. }\end{array}$ & Canada & cross-sectional & $\begin{array}{l}300 \text { immigrant and } \\
\text { refugee children }\end{array}$ & 3-13 year & $\begin{array}{c}\text { health status } \\
\text { (stunting, } \\
\text { overweight/obesity, } \\
\text { hypertension, } \\
\text { cholesterol levels, } \\
\text { health disparities) }\end{array}$ & $\begin{array}{l}\text { Dietary changes (western } \\
\text { diet); many immigrants and } \\
\text { refugees vulnerable to health } \\
\text { disparities }\end{array}$ & $\begin{array}{l}\text { 1) Refugee children are at } \\
\text { risk of stunting while } \\
\text { immigrants are more at risk } \\
\text { of overweight/obesity, } \\
\text { especially if they are older } \\
\text { and they are from privileged } \\
\text { backgrounds in low-income } \\
\text { countries; 2) } 29 \% \text { of } \\
\text { newcomer children had } \\
\text { borderline or elevated blood } \\
\text { pressure and } 52 \% \text { high } \\
\text { cholesterol levels }\end{array}$ & 14 \\
\hline
\end{tabular}


Table 2. Characteristics and main results of the included studies. [27].

\begin{tabular}{|c|c|c|c|c|c|c|c|c|c|}
\hline Author, Year & $\begin{array}{l}\text { Migrating } \\
\text { from }\end{array}$ & $\begin{array}{c}\text { Immigrating } \\
\text { to }\end{array}$ & Study Design & $\begin{array}{c}\text { N, Migrant } \\
\text { Characteristics }\end{array}$ & Age & Outcome Disease & Dietary/Cultural Issues & Main Results & QA \\
\hline $\begin{array}{l}\text { Distel, } 2019 \\
\quad[41]\end{array}$ & Mexico & USA & cohort & $\begin{array}{l}104 \text { Mexican American } \\
\text { children }\end{array}$ & $\begin{array}{c}8.39 \text { year } \\
(6-10)\end{array}$ & obesity & $\begin{array}{l}\text { Food insecurity and chronic } \\
\text { stress }\end{array}$ & $\begin{array}{c}\text { Greater food insecurity } \\
\text { associated with higher BMI } \\
\text { only when children had high } \\
\text { levels of hair cortisol }\end{array}$ & 16 \\
\hline $\begin{array}{l}\text { Buscemi, } 2011 \\
\quad[42]\end{array}$ & Latino & USA & cross-sectional & 63 Latino children & 2-17 year & obesity & $\begin{array}{l}\text { Acculturation as a moderator } \\
\text { of the relationship between } \\
\text { food insecurity and BMI: } \\
\text { higher acculturation and } \\
\text { high food security associated } \\
\text { with lower BMI }\end{array}$ & $\begin{array}{l}\text { Mean BMI percentile } \\
\text { significantly higher for } \\
\text { immigrants ( } 85 \text { ) than non } \\
\text { immigrants (70); mean BMI } \\
\text { percentile } 91 \text { for food secure } \\
\text { families and } 71 \text { for food } \\
\text { insecure (s.s.) }\end{array}$ & 14 \\
\hline $\begin{array}{l}\text { Kilanowski, } \\
2012[43]\end{array}$ & Latino & USA & cross-sectional & $\begin{array}{l}60 \text { parent-child dyads } \\
\text { of migrant } \\
\text { farmworkers }\end{array}$ & 2-13 year & overweight/obesity & $\begin{array}{l}55 \% \text { low or very low } \\
\text { household food security; } \\
\text { surprisingly, children of } \\
\text { migrants better than peers } \\
\text { concerning fruit and } \\
\text { vegetable consumption }\end{array}$ & $\begin{array}{c}22 \% \text { overweight, } 26 \% \text { obese; } \\
\text { low or very low levels of } \\
\text { food security were seen in } \\
48 \% \text { of children } \\
\text { under-normalweight, } 75 \% \\
\text { overweight, } 53 \% \text { obese }\end{array}$ & 14 \\
\hline $\begin{array}{l}\text { Ebenegger, } \\
2011 \text { [44] }\end{array}$ & $\begin{array}{c}\text { Portugal, } \\
\text { Albania/Kosovo, } \\
\text { other } \\
\text { European } \\
\text { countries; } \\
\text { Africa, Asia, } \\
\text { Latin America, } \\
\text { other }\end{array}$ & Switzerland & cross-sectional & $\begin{array}{c}542 \text { children of migrant } \\
(71 \%) \text { and non-migrant } \\
\text { parents }\end{array}$ & $5.1 \pm 0.6$ year & overweight/obesity & $\begin{array}{l}\text { Children of migrant and low } \\
\text { education level parents ate } \\
\text { more meals and snacks while } \\
\text { watching TV, more fatty } \\
\text { foods and less fruit }\end{array}$ & $\begin{array}{l}\text { Children of migrant parents } \\
\text { had higher weight, BMI and } \\
\% \text { body fat compared to } \\
\text { non-migrant; parental } \\
\text { migrant status and } \\
\text { educational level } \\
\text { independently contributed to } \\
\text { adiposity and eating habits }\end{array}$ & 14 \\
\hline $\begin{array}{l}\text { Huang, } 2018 \\
{[45]}\end{array}$ & $\begin{array}{l}\text { underdeveloped } \\
\text { area in China }\end{array}$ & $\begin{array}{l}\text { developed } \\
\text { area in China }\end{array}$ & cross-sectional & $\begin{array}{l}\text { 1154, children-caregiver } \\
\text { dyads, internal } \\
\text { migrants }\end{array}$ & 11-17 year & overweight/obesity & $\begin{array}{l}\text { Levels of acculturation } \\
\text { negatively associated with } \\
\text { overweight/obesity; children } \\
\text { with urban-to-urban migrant } \\
\text { caregivers more likely to be } \\
\text { overweight/obese than those } \\
\text { with rural-to-urban migrant } \\
\text { caregivers }\end{array}$ & $\begin{array}{l}9,7 \% \text { overweight/obese (> } \\
\text { males, > } 11-13 \text { yrs rather than } \\
14-17 \text { yrs, > urban-to-urban } \\
\text { rather than rural-to-urban) }\end{array}$ & 15 \\
\hline
\end{tabular}


Table 3. Characteristics and main results of the included studies. [27].

\begin{tabular}{|c|c|c|c|c|c|c|c|c|c|}
\hline Author, Year & $\begin{array}{l}\text { Migrating } \\
\text { from }\end{array}$ & $\begin{array}{c}\text { Immigrating } \\
\text { to }\end{array}$ & Study Design & $\begin{array}{c}\text { N, Migrant } \\
\text { Characteristics }\end{array}$ & Age & Outcome Disease & Dietary/Cultural Issues & Main Results & QA \\
\hline $\begin{array}{l}\text { Tsujimoto, } \\
2016 \text { [46] }\end{array}$ & $\begin{array}{c}\text { Mexico, } \\
\text { non-hispanic } \\
\text { white, } \\
\text { non-hispanic } \\
\text { black }\end{array}$ & USA (Boston) & cross-sectional & $\begin{array}{l}28282 \text { foreign-born and } \\
\text { US-born children }\end{array}$ & 2-19 year & overweight/obesity & Obesogenic environment & $\begin{array}{c}\text { Prevalences of } \\
\text { overweight/obesity lowest in } \\
\text { children/adolescents } \\
\text { foreign-born and who had } \\
\text { been in the US for }<1 \text { yr, } \\
\text { highest in the US-born } \\
\text { (overweight: } 23 \% \text { vs } 31.8 \% \text {, } \\
\text { obesity: } 8.2 \% \text { vs } 16.9 \% \text {, severe } \\
\text { obesity: } 2.9 \% \text { vs } 5.4 \% \text { ). Risk } \\
\text { of being overweight/obese } \\
\text { for US-born vs foreing-born } \\
\text { in the US for }<1 \text { yr: aOR } 2.2 \\
\text { overweight, aOR } 3.15 \text { obesity. }\end{array}$ & 12 \\
\hline $\begin{array}{c}\text { Iguacel, } 2018 \\
{[47]}\end{array}$ & anywhere & $\begin{array}{c}\text { Belgium, } \\
\text { Cyprus, } \\
\text { Estonia, } \\
\text { Germany, } \\
\text { Hungary, Italy, } \\
\text { Spain, } \\
\text { Sweden }\end{array}$ & cohort & $\begin{array}{c}8624 \text { children of } \\
\text { migrant }(13,4 \%) \text { and } \\
\text { non-migrant families }\end{array}$ & $\begin{array}{c}2-9.9 \text { year at } \\
\text { baseline } 4-11 \\
\text { year after } 2 \\
\text { years }\end{array}$ & overweight/obesity & $\begin{array}{c}\text { Partially explained by } \\
\text { lifestyle factors (mainly } \\
\text { sedentary habits e.g., screen } \\
\text { time) }\end{array}$ & $\begin{array}{c}\text { Overweight/obesity at T0: } \\
23.4 \% \text { migrant origin vs } \\
16.8 \% \text { native, at T1: } 28.5 \% \\
\text { migrant origin vs } 21.5 \% \\
\text { native (OR 1.3); children with } \\
\text { migrant background were } \\
\text { more likely to remain o/o } \\
\text { after } 2 \text { yrs compared to non } \\
\text { migrants (OR 1.29) }\end{array}$ & 15 \\
\hline $\begin{array}{c}\text { Labree, } 2015 \\
\text { [48] }\end{array}$ & $\begin{array}{c}\text { Turkey, } \\
\text { Morocco, } \\
\text { other western } \\
\text { and } \\
\text { not-western } \\
\text { countries }\end{array}$ & $\begin{array}{l}\text { Holland } \\
\text { (Rotterdam } \\
\quad \text { and } \\
\text { Eindhoven) }\end{array}$ & cross-sectional & $\begin{array}{l}1943 \text { immigrant and } \\
\text { native parent-child } \\
\text { dyads }\end{array}$ & $8-9$ year & overweight/obesity & $\begin{array}{l}\text { Low sleep duration, low fruit } \\
\text { and high snack intake } \\
\text { associated with higher BMIs } \\
\text { and prevalence of } \\
\text { overweight/obesity; ethnic } \\
\text { differences in sleep duration } \\
\text { and dietary intake did not } \\
\text { have a large impact on ethnic } \\
\text { differences in } \\
\text { overweight/obesity }\end{array}$ & $\begin{array}{l}\text { children of migrants had ss } \\
\text { higher BMI and higher } \\
\text { prevalence of ovw/ob, lower } \\
\text { prevalence of underweight; } \\
\text { higher intake of fruit and } \\
\text { vegetables and lower intake } \\
\text { of snacks and sweet drinks; } \\
\text { lower sleep duration. Less } \\
\text { sleep, low fruit intake, and } \\
\text { more energy-dense snack } \\
\text { consumption correlated with } \\
\text { higher BMIs and higher } \\
\text { prevalence of overweight } \\
\text { and obesity }\end{array}$ & 13 \\
\hline
\end{tabular}


Table 4. Characteristics and main results of the included studies. [27].

\begin{tabular}{|c|c|c|c|c|c|c|c|c|c|}
\hline Author, Year & $\begin{array}{c}\text { Migrating } \\
\text { from }\end{array}$ & $\begin{array}{c}\text { Immigrating } \\
\text { to }\end{array}$ & Study Design & $\begin{array}{c}\text { N, Migrant } \\
\text { Characteristics }\end{array}$ & Age & Outcome Disease & Dietary/Cultural Issues & Main Results & QA \\
\hline $\begin{array}{l}\text { Besharat Pour, } \\
2014 \text { [49] }\end{array}$ & $\begin{array}{l}\text { anywhere } \\
\text { (Africa, Asia, } \\
\text { LatinAmerica, } \\
\text { Europe } \\
\text { excluding } \\
\text { Sweden, and } \\
\text { Sweden) }\end{array}$ & Sweden & cohort & $\begin{array}{l}2589 \text { immigrant }(22 \%) \\
\text { and non-immigrant } \\
\text { children }\end{array}$ & 8 year & obesity & $\begin{array}{l}\text { Immigrants: higher } \\
\text { consumption of } \\
\text { fruit/vegetables but also of } \\
\text { cakes and sweet }\end{array}$ & $\begin{array}{l}\text { Being overweight and having } \\
\text { low physical activity more } \\
\text { common among children of } \\
\text { immigrant parents (>both } \\
\text { immigrant parents) }\end{array}$ & 13 \\
\hline $\begin{array}{c}\text { Chomitz, } 2017 \\
\text { [50] }\end{array}$ & $\begin{array}{c}\text { Asian } \\
(>\text { Chinese })\end{array}$ & $\begin{array}{c}\text { USA } \\
\text { (Chinatown in } \\
\text { Boston) }\end{array}$ & cross-sectional & $\begin{array}{l}132 \text { Asian American } \\
\text { children }\end{array}$ & $\begin{array}{l}4.9 \text { year } \\
(3.5-6)\end{array}$ & obesity & $\begin{array}{l}\text { Perceived parents' barriers: } \\
\text { 1) worry about safety when } \\
\text { child plays outside 2) healthy } \\
\text { food too expensive }\end{array}$ & $\begin{array}{l}32.6 \% \text { overweight/obese (vs } \\
23.4 \% \text { overall USA and } 9 \% \\
\text { Asians) - children of less } \\
\text { acculturated parents, more } \\
\text { likely with lower income or } \\
\text { recent immigrants, were } 3,5 \\
\text { times more likely to be } \\
\text { overweight/obese than those } \\
\text { of more acculturated parents } \\
\text { - more acculturated more } \\
\text { likely to provide fruits daily } \\
\text { or more but also more sugary } \\
\text { snacks }\end{array}$ & 14 \\
\hline $\begin{array}{c}\text { Alasagheirin, } \\
2018 \text { [51] }\end{array}$ & Sudan & USA & cross-sectional & $\begin{array}{l}64 \text { immigrant and } \\
\text { refugee Sudanese } \\
\text { children }\end{array}$ & 5-18 year & $\begin{array}{l}\text { growth, body } \\
\text { composition, } \\
\text { metabolic risk, } \\
\text { physical activity and } \\
\text { food security }\end{array}$ & $\begin{array}{l}\text { Food insecurity in } 40 \% \text { of } \\
\text { families, sedentary habits } \\
\text { reported by many }\end{array}$ & $\begin{array}{l}32 \% \text { obese, } 46 \% \text { low lean } \\
\text { mass; high cholesterol } 23 \% \text {, } \\
\text { high triglycerides } 32 \%, \text { high } \\
\text { insulin resistance } 15 \% \\
\text { (correlates with high risk of } \\
\text { diabetes and cardiovascular } \\
\text { problem); Low bone mass } \\
\text { which could contribute to } \\
\text { osteoporosis }\end{array}$ & 13 \\
\hline $\begin{array}{c}\text { Tovar, } 2012 \\
\text { [52] }\end{array}$ & $\begin{array}{l}\text { Haiti, Latin } \\
\text { America, } \\
\text { Brazil }\end{array}$ & USA & $\begin{array}{l}\text { randomized } \\
\text { controlled trial }\end{array}$ & 383 mother-child dyads & $\begin{array}{l}\text { mothers } 20-55 \\
\text { year, children } \\
3-12 \text { year }\end{array}$ & overweight/obesity & $\begin{array}{l}\text { A low demanding/high } \\
\text { responsive feeding style is } \\
\text { significantly and positively } \\
\text { associated with higher child } \\
\text { weight. }\end{array}$ & $\begin{array}{c}72 \% \text { of mothers and } 43 \% \text { of } \\
\text { children overweight/obese. } \\
\text { Fifteen percent of mothers } \\
\text { reported their feeding style } \\
\text { as being high } \\
\text { demanding/high responsive; } \\
32 \% \text { as being high } \\
\text { demanding/low responsive; } \\
34 \% \text { as being low } \\
\text { demanding/high responsive } \\
\text { and } 18 \% \text { as being low } \\
\text { demanding/low responsive. }\end{array}$ & 18 \\
\hline
\end{tabular}


Table 5. Characteristics and main results of the included studies. [27].

\begin{tabular}{|c|c|c|c|c|c|c|c|c|c|}
\hline Author, Year & $\begin{array}{c}\text { Migrating } \\
\text { from }\end{array}$ & $\begin{array}{c}\text { Immigrating } \\
\text { to }\end{array}$ & Study Design & $\begin{array}{c}\text { N, Migrant } \\
\text { Characteristics }\end{array}$ & Age & Outcome Disease & Dietary/Cultural Issues & Main Results & QA \\
\hline $\begin{array}{c}\text { Cheah, } 2012 \\
\text { [53] }\end{array}$ & China, Korea & USA & cross-sectional & $\begin{array}{c}130 \text { children of } \\
\text { first-generation } \\
\text { immigrants from China } \\
(62 \%) \text { and Korea }(38 \%)\end{array}$ & $3-8$ year & obesity & $\begin{array}{l}\text { Parents' food insecurity in } \\
\text { their childhood associated } \\
\text { with obesity-promoting } \\
\text { behaviors and outcomes }\end{array}$ & $\begin{array}{c}20 \% \text { overweight/obese; } \\
\text { parents with food insecurity } \\
\text { in childhood: } 1 \text { ) evaluated } \\
\text { their children weighing less } \\
\text { than ideal 2) allowed more } \\
\text { servings of soda and sweets; } \\
\text { early life material } \\
\text { deprivation: 1) less concern } \\
\text { about children's diets, 2) less } \\
\text { concern child eating too } \\
\text { much or becoming } \\
\text { overweight }\end{array}$ & 14 \\
\hline $\begin{array}{c}\text { Cook, } 2017 \\
\text { [54] }\end{array}$ & Asia & $\begin{array}{c}\text { USA } \\
\text { (California) }\end{array}$ & cohort & $\begin{array}{l}1525 \text { Asian American } \\
\text { adolescents }\end{array}$ & $12-17$ year & overweight/obesity & $\begin{array}{l}\text { The two lifestyle factors (i.e., } \\
\text { physical activity and fast } \\
\text { food consumption) were not } \\
\text { associated with } \\
\text { overweight/obesity }\end{array}$ & $\begin{array}{l}\text { Overweight/obesity rate } \\
\text { higher among those with } \\
\text { lower }(24.7 \%) \text { than higher } \\
\text { (13.4\%) family incomes; } \\
\text { higher among those in the } \\
\text { low-SES (29.0\%) than } \\
\text { middle/high-level SES ethnic } \\
\text { groups (11.6\% and } 12.8 \% \text {, } \\
\text { respectively). By ethnicity, } \\
\text { overweight/obesity lowest } \\
\text { among Japanese (4.8\%) and } \\
\text { highest among Filipino } \\
\text { (26.3\%) and Southeast Asians } \\
\text { (25.5\%). Adolescents in } \\
\text { high-middle SES were far } \\
\text { less likely to be overweight } \\
\text { or obese than those in low } \\
\text { SES: this was more } \\
\text { pronounced for foreign-born } \\
\text { adolescents vs US-born. }\end{array}$ & 14 \\
\hline $\begin{array}{c}\text { Zulfiqar, } 2018 \\
{[55]}\end{array}$ & $\begin{array}{l}\text { HIC and } \\
\text { LMIC }\end{array}$ & Australia & cross-sectional & $\begin{array}{c}4115 \text { children } \\
\text { originating from } \\
\text { Australia }(58 \%), \text { HIC } \\
(30 \%), \text { LMIC }(12 \%)\end{array}$ & 4-11 year & overweight/obesity & $\begin{array}{l}\text { Higher intake of both } \\
\text { vegetables and } \\
\text { sugar-sweetened-beverages, } \\
\text { higher inclination toward } \\
\text { sedentary activities and } \\
\text { lower organized sports } \\
\text { participation. }\end{array}$ & $\begin{array}{l}\text { Higher overweight/obesity } \\
\text { rates in immigrants from } \\
\text { LMIC (LMIC/HIC/Australian } \\
\% \text { : boys 30/23/22, girls } \\
\text { 35/22/24) and higher in } \\
\text { second-generation }\end{array}$ & 15 \\
\hline
\end{tabular}


Table 6. Characteristics and main results of the included studies. [27].

\begin{tabular}{|c|c|c|c|c|c|c|c|c|c|}
\hline Author, Year & $\begin{array}{l}\text { Migrating } \\
\text { from }\end{array}$ & $\begin{array}{c}\text { Immigrating } \\
\text { to }\end{array}$ & Study Design & $\begin{array}{c}\text { N, Migrant } \\
\text { Characteristics }\end{array}$ & Age & Outcome Disease & Dietary/Cultural Issues & Main Results & QA \\
\hline $\begin{array}{l}\text { Iriart, } 2011 \\
\quad[56]\end{array}$ & Hispanic & USA & cross-sectional & $\begin{array}{l}3102 \text { Hispanic }(38 \%) \\
\text { and non-Hispanic } \\
\text { children }\end{array}$ & 2-19 year & stunting & $\begin{array}{l}\text { Hispanics more likely to be } \\
\text { less than full food secure } \\
\text { (30.5\% vs } 11.8 \%) \text {; hispanics } \\
\text { with normal weight were } \\
\text { more likely to be fully food } \\
\text { secure }\end{array}$ & $\begin{array}{c}\text { Hispanics: highest } \\
\text { proportion of stunting }(6.6 \%), \\
\text { overweight/obesity (39.3\%), } \\
\text { stunting among normal } \\
\text { weight (7\%), stunting among } \\
\text { overweight/obese (6\%) } \\
\text { compared to non-hispanic } \\
\text { whites, non-hispanic blacks, } \\
\text { other races; tendency for } \\
\text { overweight/obesity in } \\
\text { hispanic children who face } \\
\text { adverse socioeconomic } \\
\text { conditions to experience a } \\
\text { higher prevalence of stunting }\end{array}$ & 15 \\
\hline $\begin{array}{l}\text { Choudhary, } \\
2009[57]\end{array}$ & $\begin{array}{l}44 \% \text { rural, } \\
51 \% \text { urban }\end{array}$ & Mumbai city & cross-sectional & $\begin{array}{l}481 \text { internal migrant } \\
\text { children }\end{array}$ & $<3$ year & $\begin{array}{l}\text { stunting, } \\
\text { underweight, wasting }\end{array}$ & poverty & $\begin{array}{l}\text { Stunting and low } \\
\text { mother-BMI >in migrants } \\
\text { with high disadvantage for } \\
\text { rural migrants; as the years } \\
\text { in Mumbai increase, } \\
\text { migrants learn to assimilate } \\
\text { to the new environment and } \\
\text { the disadvantage compared } \\
\text { to non-migrants declines }\end{array}$ & 15 \\
\hline Lee, 2015 [58] & North Korea & South Korea & cohort & 70 immigrant children & 6-15 year & stunting and obesity & $\begin{array}{l}\text { Those who lived in South } \\
\text { Korea longer were less likely } \\
\text { to be currently stunted }\end{array}$ & $\begin{array}{l}\text { At entry } 11.4 \% \text { stunted and } \\
\text { only } 5.7 \% \text { after } 2 \text { years. The } \\
\text { prevalence of obesity was } \\
\text { similar to that of SK children. } \\
\text { The likelihood of remaining } \\
\text { stunted was significantly } \\
\text { associated with older age } \\
\text { and shorter residency in SK. } \\
\text { The was no significant } \\
\text { association with food } \\
\text { security situation at birth. }\end{array}$ & 14 \\
\hline $\begin{array}{l}\text { Werneck, } 2008 \\
\quad[59]\end{array}$ & $\begin{array}{c}\text { Portugal, } \\
\text { Brazil, Angola, } \\
\text { Mozambique, } \\
\text { Azores }\end{array}$ & $\begin{array}{l}\text { Canada } \\
\text { (Toronto) }\end{array}$ & case-control & 104 immigrant children & $\leq 48$ months & $\begin{array}{l}\text { Early childhood } \\
\text { caries }\end{array}$ & $\begin{array}{l}\text { Immigrants have difficulty in } \\
\text { obtaining dental care } \\
\text { primarily; factors that } \\
\text { correlated the most with } \\
\text { early childhood caries were } \\
\text { family without dental } \\
\text { insurance, lack of a family } \\
\text { dentist, and frequency of } \\
\text { snack consumption. }\end{array}$ & $35 \%$ early childhood caries & 15 \\
\hline
\end{tabular}


Table 7. Characteristics and main results of the included studies. [27].

\begin{tabular}{|c|c|c|c|c|c|c|c|c|c|}
\hline Author, Year & $\begin{array}{l}\text { Migrating } \\
\text { from }\end{array}$ & $\begin{array}{c}\text { Immigrating } \\
\text { to }\end{array}$ & Study Design & $\begin{array}{c}\text { N, Migrant } \\
\text { Characteristics }\end{array}$ & Age & Outcome Disease & Dietary/Cultural Issues & Main Results & QA \\
\hline $\begin{array}{l}\text { Vatanparast, } \\
2013[60]\end{array}$ & $\begin{array}{c}\text { Asia, Africa, } \\
\text { Middle East } \\
\text { and Latin } \\
\text { America }\end{array}$ & Canada & cross-sectional & $\begin{array}{l}72 \text { children ( } 33 \\
\text { immigrant and } 39 \\
\text { refugee) }\end{array}$ & 6-11 year & vitamin D deficiency & $\begin{array}{l}\text { Calcium intake inadequacy } \\
74 \% \text { in migrants and } 77 \% \text { in } \\
\text { refugees, vitamin D intake } \\
\text { inadequacy } 81 \% \text { in migrants } \\
\text { and } 97 \% \text { in refugees }\end{array}$ & $\begin{array}{l}\text { Serum vitamin } \mathrm{D} \\
\text { deficiency/inadequacy in } 63 \% \\
\text { of migrants and } 80 \% \text { of } \\
\text { refugees }\end{array}$ & 12 \\
\hline $\begin{array}{l}\text { Sacri, } 2017 \\
\text { [61] }\end{array}$ & anywhere & France & cross-sectional & $\begin{array}{c}657 \text { immigrant }(14 \%) \\
\text { and non-immigrant } \\
(86 \%) \text { children }\end{array}$ & 3.9 year $(<6)$ & iron deficiency & $\begin{array}{l}\text { Male gender, mother being a } \\
\text { migrant, underprivileged } \\
\text { family status and low } \\
\text { maternal education were } \\
\text { related to ID }\end{array}$ & $\begin{array}{l}\text { Significantly associated with } \\
\text { mother being a migrant: low } \\
\text { serum ferritinemia ( } 32.1 \\
\text { mcg/L vs } 44.9 \text { ) and iron } \\
\text { deficiency }(7 \% \text { vs } 2 \%) ; \text { iron } \\
\text { deficiency prevalence } 2.8 \% \text { to } \\
3.2 \% \text { depending on serum } \\
\text { ferritin threshold of } 10 \text { or } \\
12 \mu \mathrm{L} / \mathrm{L} .\end{array}$ & 15 \\
\hline $\begin{array}{l}\text { Saunders, } \\
2016[62]\end{array}$ & $\begin{array}{c}\text { from } \\
\text { industrialized } \\
\text { and not } \\
\text { industrialized } \\
\text { countries } \\
\text { (especially } \\
\text { from Europe) }\end{array}$ & Canada & cross-sectional & $\begin{array}{l}2614 \text { children }(47.6 \% \\
\text { immigrant) }\end{array}$ & $12-72$ month & iron deficiency & $\begin{array}{l}\text { Younger age, male sex, high } \\
\text { cow's milk intake, longer } \\
\text { breastfeeding duration } \\
\text { associated with lower serum } \\
\text { ferritin }\end{array}$ & $\begin{array}{l}\text { No association between } \\
\text { family immigrant status and } \\
\text { iron status, no need for iron } \\
\text { supplementation in } \\
\text { immigrants' children }\end{array}$ & 15 \\
\hline Kim, 2006 [63] & Latin America & $\begin{array}{l}\text { USA (Rural } \\
\text { Nebraska) }\end{array}$ & cohort & $\begin{array}{l}29 \text { Latino immigrant } \\
\text { children }\end{array}$ & $4-8$ year & $\begin{array}{l}\text { micronutrients } \\
\text { deficiency (plasma } \\
\text { concentrations of } \\
\text { vitamin } \mathrm{E}, \text { vitamin } \mathrm{C} \text {, } \\
\text { selenium and } \\
\text { carotenoids) }\end{array}$ & $\begin{array}{l}59 \% \text { reported consuming less } \\
\text { than the Estimated Average } \\
\text { Requirement for vitamin E; } \\
\text { in general, consumption of } \\
\text { the Recommended Dietary } \\
\text { Allowances for vitamin C } \\
\text { and selenium }\end{array}$ & $\begin{array}{l}69 \% \text { vitamin E inadequacy; } \\
\text { in general, normal levels of } \\
\text { vitamin C and selenium. }\end{array}$ & 13 \\
\hline
\end{tabular}

SES: socioeconomic status; HIC: high-income countries; LMIC: low-middle-income countries; QA: quality assessment according to the EPHPP. 
Table 8. Quality assessment of the included studies according to the EPHPP [27].

\begin{tabular}{|c|c|c|c|c|c|c|c|}
\hline Author, Year & $\begin{array}{c}\text { Study } \\
\text { Design }\end{array}$ & Blinding & $\begin{array}{l}\text { Selection } \\
\text { Bias }\end{array}$ & $\begin{array}{c}\text { Data } \\
\text { Collection }\end{array}$ & Confounding & $\begin{array}{c}\text { Withdrawal } \\
\text { and Drop-Outs }\end{array}$ & $\begin{array}{l}\text { Overall } \\
\text { Rating }\end{array}$ \\
\hline Rosas 2010 [35] & 1 & 2 & 3 & 3 & 3 & 1 & 13 \\
\hline Kaiser 2015 [36] & 1 & 2 & 3 & 3 & 3 & 2 & 14 \\
\hline Kobel 2017 [37] & 2 & 2 & 3 & 3 & 2 & 2 & 14 \\
\hline Early 2019 [39] & 1 & 2 & 3 & 3 & 2 & 1 & 12 \\
\hline Geremia 2015 [40] & 1 & 2 & 2 & 3 & 2 & 2 & 12 \\
\hline Lane 2018 [22] & 1 & 2 & 3 & 3 & 3 & $2 ?$ & 14 \\
\hline Distel 2019 [41] & 2 & 2 & 3 & 3 & 3 & 3 & 16 \\
\hline Buscemi 2011 [42] & 1 & 2 & 3 & 3 & 3 & 2 & 14 \\
\hline Kilanowski 2012 [43] & 1 & 2 & 3 & 3 & 3 & 2 & 14 \\
\hline Ebenegger 2011 [44] & 1 & 2 & 3 & 3 & 3 & 2 & 14 \\
\hline Huang 2018 [45] & 1 & 2 & 3 & 3 & 3 & 3 & 15 \\
\hline Tsujimoto 2016 [46] & 1 & 2 & 3 & 3 & 2 & 1 & 12 \\
\hline Iguacel 2018 [47] & 1 & 2 & 3 & 3 & 3 & 3 & 15 \\
\hline Labree 2015 [48] & 1 & 2 & 2 & 3 & 2 & 3 & 13 \\
\hline Besharat Pour 2014 [49] & 1 & 2 & 3 & 3 & 2 & 2 & 13 \\
\hline Chomitz 2017 [50] & 1 & 2 & 3 & 3 & 2 & 3 & 14 \\
\hline Alasagheirin 2018 [51] & 1 & 2 & 3 & 3 & 2 & 3 & 13 \\
\hline Tovar 2012 [52] & 3 & 3 & 3 & 3 & 3 & 3 & 18 \\
\hline Cheah 2012 [53] & 1 & 2 & 3 & 3 & 3 & 2 & 14 \\
\hline Cook 2017 [54] & 1 & 2 & 3 & 3 & 3 & 2 & 14 \\
\hline Zulfiqar 2018 [55] & 2 & 2 & 3 & 3 & 2 & 3 & 15 \\
\hline Iriart 2011 [56] & 1 & 2 & 3 & 3 & 3 & 3 & 15 \\
\hline Choudhary 2009 [57] & 2 & 2 & 3 & 3 & 2 & 3 & 15 \\
\hline Lee 2015 [58] & 2 & 2 & 3 & 3 & 2 & 2 & 14 \\
\hline Werneck 2008 [59] & 2 & 3 & 3 & 3 & 3 & 1 & 15 \\
\hline Vatanparast 2013 [60] & 1 & 2 & 3 & 3 & 2 & 1 & 12 \\
\hline Sacri 2017 [61] & 1 & 2 & 3 & 3 & 3 & 3 & 15 \\
\hline Saunders 2016 [62] & 1 & 2 & 3 & 3 & 3 & 3 & 15 \\
\hline Kim 2006 [63] & 1 & 2 & 3 & 3 & 2 & 2 & 13 \\
\hline
\end{tabular}

\subsection{Evidence Synthesis}

\subsubsection{Overweight, Obesity, and Associated Complications}

Evidence for this outcome comes from 21 papers: 13 cross-sectional studies, 5 cohort studies, 3 randomized controlled trials. Quality was determined "good" for 18 of them, and only "fair" for 3 studies. Twelve studies were conducted in the USA, the rest were performed in Canada, Brazil, China, Australia, and several countries in Europe. The studies included different variables such as SES, acculturation, ethnicity, eating habits, lifestyles including physical activity and sleeping hours, thus making the studies extremely heterogeneous.

Many studies reported a high prevalence of overweight/obesity among immigrant children.

A binational study (Mexico and California, USA) performed on 603 mother-child dyads by Rosas et al. [35] found a significantly higher prevalence of obesity among the children of Mexican descent who were born in California compared to those who were born in Mexico ( $53.3 \%$ vs $14.9 \% ; p<0.01$ ). Maternal obesity was a determinant of childhood obesity in both samples, but it was significantly higher in California (49\%) compared to Mexico (33\%; $p<0.01)$. Although the link between maternal obesity and child's weight status may suggest genetic or epigenetic factors, it probably also strongly reflects cultural and environmental characteristics shared with the child. 
The paper of Kaiser et al. [36] examined the influence of age and gender on food patterns of Latino children in rural communities in California. $51 \%$ of the total sample was overweight or obese, with no significant effect of neither age nor gender on food patterns; mother' s acculturation level was positively related $(p=0.0002)$ to children consumption of less healthy foods. This contradicts the findings of Rosas on the basis of quantifiable weight, although, it can be argued that certain parameters, not studied, including mental well-being, discrimination, etc. due to, or as a result of, an inability to adapt or resistance to adapt, may be looked into.

In a study by Kobel et al. [37] performed on 525 school-aged children in Germany, which analyzed the effects of interventions to promote healthier diet and behaviors, the prevalence of overweight and obesity in those with a migration background was $11.8 \%$ and obesity alone $5.7 \%$ (vs $9 \%$ and $4 \%$, respectively in German children [38]).

Also, the study by Early et al. [39] investigated the efficacy of an intervention aimed at improving health behaviors in 68 children (mean age 10.8 years) from low-income families enrolled from 3 clinic sites in California, USA. Most of the children had a migration background with $85 \%$ being of Hispanic origin and 3\% Black/African American. The reported incidence of overweight was $11.8 \%$ and that of obesity $54.4 \%$, very similar to the figures reported by the previously cited research from the USA.

Another cross-sectional study by Geremia et al. [40] showed a high prevalence of being overweight and obese (16.3\% (95\% CI: 13.3-19.3) and 8.3\% (95\% CI: 6.1-10.5)) in schoolchildren of a southern Brazilian city with a strong Italian immigration influence. Factors such as omission of breakfast (OR 0.669 (95\% CI: 0.499-0.896); $p=0.007$ ), overweight and obesity in mothers (OR 1.022 (95\% CI: 1.012-1.031); $p<0.001$ ), age (OR 0.875 (95\% CI: 0.776-0.987); $p=0.030$ ) and male gender (OR 1.341 (95\% CI: $1.009-1.782) ; p=0.043)$ were significantly associated with excess weight.

Lane et al. [22] analyzed the health status of 300 immigrant and refugee children aged 3-13 years who had been in Canada for less than 5 years. The results indicated that older immigrant children (OR 1.18 (95\% CI: 1.03-1.35); $p=0.017$ ), mainly those from more privileged backgrounds, who coincidingly consumed a poorer-quality diet (OR 0.94 (95\% CI: 0.90-0.99); $p=0.020)$, were at a higher risk of overweight and obesity (compared to refugees, who had a higher risk of stunting ( $23 \%$ vs $4.6 \%$; $p<0.05)$ ). Moreover, $52 \%$ of the newcomer children had high cholesterol levels and $29 \%$ had borderline or elevated blood pressure, higher rates compared to what reported for Canadian children $(35 \%$ and $7 \%$ respectively).

Several studies analyzed the relationship between obesity and FI with conflicting results.

The study by Distel et al. [41] considered the relationship between FI and chronic stress on BMI in 104 Mexican-American children aged 6-10 years. Greater food insecurity was associated with higher BMI only when children had high levels of hair cortisol ( $b e t a=3.50, p=0.02)$, highlighting the great influence of stress on the development of overweight and obesity.

Buscemi et al. [42] confirmed a high prevalence of overweight and obesity among Latino children in the US: those with both immigrant parents had a higher $(p=0.03)$ mean BMI percentile (85.05, $\mathrm{SD}=18.72)$ compared to those with non-immigrant parents $(70.30, \mathrm{SD}=32.84)$. Furthermore, the mean BMI percentile of children from food secure families was significantly higher than that of children from food insecure families $(p=0.022)$, with acculturation being a significant moderator of the relationship between food insecurity and BMI. In fact, high acculturation and high food security scores were associated with lower BMI $(p=0.003)$.

Kilanowski et al. [43] published a cross-sectional study reporting that, among migrant farm-worker families in the US, low or very low levels of food security were seen in $48 \%$ of children with under or normal weight, $75 \%$ in overweight and $53 \%$ in obese children. However, differently from Buscemi and colleagues [42], no statistical significance was found between acculturation and BMI.

The role of acculturation on the development of childhood obesity in migrant children was also explored by other authors.

In a Swiss multidisciplinary lifestyle intervention study by Ebenegger et al. [44] preschooler children of migrant parents of low education backgrounds had an increase in adiposity $(p \leq 0.04)$ 
and media use $(p \leq 0.005)$ and less healthy habits (more consumption of fatty foods and less of fruit $(p \leq 0.0001)$, vegetables and water $(p \leq 0.03))$ compared to those with non-migrant or medium-high educational level parents.

The study by Huang et al. [45] assessed the association between acculturation and body weight among the children (11-17 years) of internal migrants in China. The overall prevalence of overweight/obesity was $12.5 \%$ in boys and $6.1 \%$ in girls; migrant children who had urban-to-urban migrant caregivers were more likely to be overweight/obese than those with rural-to-urban migrant caregivers (OR 1.80 (95\% CI: 1.13-2.86); $p=0.014)$, and those with caregivers having higher levels of acculturation (OR 0.99 (95\% CI: 0.98-1.00); $p=0.078$ ) were less likely to be overweight/obese than those with lower levels. However, there was no statistically significant difference in terms of acculturation score between overweight/obese and normal weight migrant children.

The effect of the length of stay in the new country was analyzed by two papers. Using data from the National Health and Nutrition Examination Survey, a cross-sectional study by Tsujimoto et al. [46] assessed the correlation between the length of time in the US and the prevalence of obesity in 28282 children and adolescents. The prevalence of overweight/obesity was lowest in those who were foreign-born and had been in the US for less than 1 year compared to the US-born (overweight or more: $23 \%$ vs $31.8 \%$, obesity: $8.2 \%$ vs $16.9 \%$, severe obesity: $2.9 \%$ vs $5.9 \%$ ), with an increasing trend in foreign-born children who lived in US for more than 1 year (overweight or more: adjOR 1.38 (95\% CI: 0.85-2.24), obesity: adjOR 1.80 (95\% CI: 0.82-3.96), severe obesity: adjOR 1.36 (95\% CI: $0.25-7.30)$ ), suggesting that foreign-born children and adolescents are susceptible to the obesogenic environment of the USA with unhealthy dietary habits (high energy, sugar, and fat intake).

In the cross-sectional study by Iguacel et al. [47] about the association between social vulnerabilities and children' s weight status in Europe, children with a migrant background were more likely to be overweight/obese at the first evaluation (OR 1.30 (99\% CI: 1.04-1.62); $p=0.003$ ) and to remain so after 2 years (OR 1.14 (99\% CI: 0.85-1.52); $p=0.239$ ) compared to non-migrants. In this study the association between social vulnerabilities and children's weight was only partially explained by lifestyle factors, maternal BMI and socioeconomic status indicators. Indeed, children who accumulated more vulnerabilities (migrant background, parents unemployed, non-traditional families, lack of a social network) did not show a higher likelihood of being overweight o underweight (OR 1.33 (99\% CI: 0.91-1.94)).

Other studies investigated the role of healthy behaviors (physical activity, diet, and sleep quality) in the progression towards being overweight or obese in children with migrant backgrounds.

Labree et al. [48] reported a cross-sectional study including 1943 parent-child native Dutch and immigrant dyads. Children of migrant descent had higher BMIs and prevalence of overweight and obesity $(p<0.05)$; they had lower physical activity levels and lower sleep duration, but a higher fruit and vegetable intake and a lower consumption of sugar-sweetened beverages and energy-dense snacks (all $p<0.05$ ). Less sleep, low fruit intake, and more energy-dense snack consumption correlated with higher BMIs and higher prevalence of being overweight and obese (all $p<0.05$ ). Ethnic differences between native Dutch and migrant children only slightly contributed to explaining differences in the prevalence of being overweight and obese.

Also, the Swedish study by Besharat Pour et al. [49] assessed the nutritional status, level of physical activity, and overweight/obesity among children of immigrant or Swedish-born parents. Children of migrants were more likely to be overweight (OR 1.33 (95\% CI: 1.07-1.66)), have low physical activity (OR 1.30 (95\% CI: 1.05-1.62)) and have parents with the lowest education level compared to children of Swedish parents. However, children of immigrants complied more fully with nutritional recommendations compared to the Swedish (OR = 1.31 (95\% CI 1.08-1.58]), even if they had a lower intake of micronutrients such as vitamins A (mean $1049.9 \mathrm{ug} /$ day, $\mathrm{SD}=366.6$ ) and D (mean $5.1 \mathrm{ug} / \mathrm{day}$, $\mathrm{SD}=1.8$ ), calcium (mean $1162.8 \mathrm{mg} /$ day, $\mathrm{SD}=348.6$ ) and iron (mean $10.57 \mathrm{mg} /$ day, $\mathrm{SD}=1.83$ ), and a higher consumption of sugar and sweets $(p<0.01)$. 
Chomitz et al. [50] analyzed the associations between diet, physical activity and acculturation among Chinese American children. The prevalence of overweight and obesity, as reported by the parents, was higher (32\%) among children of less acculturated parents, who were 3.5 times more likely to be overweight/obese than those from most acculturated families. However, after adjustment by age, gender and income proxy, acculturation status amongst migrant children was not a significant predictor of the child health outcomes.

The cross-sectional study by Alasagheirin et al. [51] highlighted some potential risks related to body composition and metabolic features in 64 Sudanese children living in the US: $26.6 \%$ were overweight or obese determined by BMI and $28 \%$ were obese determined by body fat percent; levels of cholesterol were borderline/high in $23.4 \%$, triglycerides in $32.8 \%$, and $16 \%$ had risk levels for insulin resistance. Food insecurity was reported by $40 \%$ of the families; moreover, the levels of physical activity in Sudanese children were very low and $42.2 \%$ of them spent at least $3 \mathrm{~h}$ a day watching television or using electronics devices.

Parental feeding styles may influence children's eating habits and consequently their weight. This topic is highlighted by Tovar et al. [52] in a randomized controlled lifestyle intervention study that included 383 mother-child dyads of Brazilian, Haitian, and Latino peoples living in the US. 72\% of mothers and $43 \%$ of the children were overweight and obese. A low demanding/high responsive feeding style in immigrant mothers (few rules, children were allowed much freedom) was significantly and positively associated with child weight status, even after adjusting for ethnicity, acculturation and stress (beta $=0.56, p=0.01$ ).

The relationship between immigrant parents' early life deprivation and child feeding practices and weight is treated by Cheah et al. [53]. Korean and Chinese immigrant parents of children aged 3-8 years in the US were interviewed about food insecurity, acculturation, child feeding practices, evaluations whether their child weight was more or less than the ideal and child consumption of soda drink and candy; BMI was calculated for parents and children. Even if only $20 \%$ of immigrant children were overweight or obese according to BMI, parents' FI in their childhood was associated with obesity-promoting behaviors and outcomes (beta $=0.17, p<0.05$ ).

The correlation between socioeconomic status and overweight/obesity was treated by Cook et al. [54] among seven Asian American ethnic groups. Adolescents from migrant families in the high-middle-level socioeconomic status ethnic group were less likely to be obese/overweight than those in the low-level $(p<0.01)$; this feature was not significant among US-born adolescents.

The cross-sectional analysis by Zulfiqar et al. [55] confirmed a higher trend of overweight/obesity in Australia for immigrant children from low-middle income countries (boys: 30\%, 23\%, 22\%; girls: $35 \%, 22 \%, 24 \% ; p=0.002$; for low-middle income countries, Australian-born and high-income countries respectively). The results show that the excess weight/obesity risk in this group is primarily due to sedentary activities $(p<0.01)$.

\subsubsection{Stunting}

In agreement with WHO definitions, stunting was defined as height-for-age-and-sex less than two standard deviations below the median.

Evidence for this outcome comes from 5 papers: 4 cross-sectional studies and 1 cohort study. Quality was classified as good for all of them. The majority of the studies assessed a remarkable stunting prevalence in migrant children, highlighting the need for specific screening and therapeutic programs. Alasagheirin et al. [51] conducted a cross-sectional descriptive study of skeletal growth in 64 Sudanese children aged 5-18 living in the US and found that almost 5\% (4.69\%) of them were abnormally slowed in growth, and girls were nearly twice as likely to be stunted than boys (6.06\% vs. $3.23 \%$, respectively).

Similarly, the study by Lane et al. [22] documented that the prevalence of stunted growth was $4.6 \%$ in 300 migrant children aged 3-13 years who had been in Canada for less than 5 years. 
In 2011 Iriart et al. [56] investigated the prevalence of stunting in Hispanic children living in the US. Among 3102 children and adolescents aged 2-19 years, the prevalence of stunting was significantly higher $(p<0.001)$ in Hispanics $(6.6 \%)$ than in non-Hispanic whites $(2.2 \%)$. Normal weight foreign-born Hispanic children who had been in the US 5 years or more experienced the greatest prevalence of stunting, significantly higher than their counterparts who had been in the US for less than 5 years $(25.7 \%$ vs. $11.9 \%, p<0.01)$. Stunting prevalence did not significantly differ between normal weight and overweight/obese Hispanic children, but overweight/obese children with economic difficulties experienced a higher prevalence of stunting, compared to those who do not face adverse socio-economic conditions $(p<0.10)$. The proportion of stunting differed significantly by country of birth $(14.1 \%$ for foreign-born vs 5.2\% for US-born; $p<0.001)$, language of family interview ( $9.4 \%$ for Spanish vs. 5.6\% for English; $p<0.05)$, and number of people in the family $(10.5 \%$ for 6 or more members, $3.1 \%$ for $4-5$ members, $7.7 \%$ for $1-3$ members; $p<0.001)$. Moreover, also low educational level $(p<0.10)$ and low family income $(p<0.10)$ were found to be associated with stunting.

Choudhary et al. [57] assessed the prevalence of stunting in children below 3 years of age migrating to Mumbai from rural and urban areas of India. This paper showed a significantly higher incidence of stunting among rural migrants compared to urban ones $(p<0.05)$ and among migrants who came to Mumbai during the last five years compared to migrants who had been staying in Mumbai for longer $(p<0.05)$, supporting a positive effect of the length of residency on the level of malnutrition due to the migrants' capacity of adaptation into the new environment.

Evidence supporting a correlation between migration and stunting was also confirmed by Lee et al. [58], who analyzed 70 North Korean children between 6-15 years migrating to South Korea. Stunting, evaluated using the Korean Growth Chart, was significantly higher $(p=0.040)$ in North Korean children at the time of entry to South Korea $(11.4 \%)$ than in South Korean children (1.0\%), but interestingly, after an average length of stay of 2 years the prevalence of stunting decreased in migrating children $(5.7 \%)$, but North Korean children were still shorter $(p=0.000)$, probably because linear growth deficits are slow to recover as overall nutritional status remains poor compared to peers without exposures to FI.

\subsubsection{Early Childhood Caries}

We found one good-quality, case-control study by Werneck et al. [59] evaluating the prevalence and risk factors of early childhood caries (ECC) in Portuguese-speaking children aged 48 months or younger and with at least one parent from Portugal, Brazil, Angola, Mozambique and the Azores migrating to Canada. One-third (35\%) of the children recruited had ECC. The factors that correlated the most with ECC were family without dental insurance (adjOR 4.87 (95\% CI: 1.85-12.82); $p=0.001$ ), lack of a family dentist (adjOR 3.96 (95\% CI: 1.34-11.70); $p=0.013$ ), and frequency ( $\geq 2$ vs $<2$ ) of snack consumption (adjOR 3.79 (95\% CI: 1.32-10.83); $p=0.013$ ). Subjects from low-income families were almost 4 times more likely to have ECC, and low parental knowledge of harmful feeding habits were linked to higher prevalence of ECC (OR 2.84 (95\% CI: 1.27-6.33); $p=0.010)$. Also, parents' countries of origin and their age at the time of immigration were significantly associated with ECC: children of parents from non-European countries migrating to Canada had a higher occurrence of ECC $(p<0.026)$, and parents who migrated in their 20s or older were 2-4 times more likely to have a child with ECC than those who immigrated at younger age $(p<0.04)$. A person who immigrates when he or she is a child would be more exposed to positive oral health messages at school, and this is not the case for someone who immigrated as an adult. Surprisingly, oral hygiene history was not a factor influencing dental health.

\subsubsection{Micronutrient Deficiency}

Evidence for this outcome comes from 3 cross-sectional and 1 cohort studies. Quality was classified as good for 3 of them and fair for one. These studies reported a high prevalence of micronutrient deficiency in migrant children. 
Vitamin D deficiency was evaluated by Vatanparast et al. [60], distinguishing between migrant and refugee children aged 6-11 years in Canada. They noted a mean serum 25 hydroxyvitamin $\mathrm{D}(25(\mathrm{OH}) \mathrm{D})$ concentration significantly higher in non-immigrant children than in immigrant or refugees, with overall a vitamin D deficiency/inadequacy in $63 \%$ of migrants and $80 \%$ of refugees. The prevalence of calcium intake inadequacy was $74 \%$ in migrants and $77 \%$ in refugees, and of vitamin D intake inadequacy $81 \%$ in migrants and $97 \%$ in refugees. Female sex (Standardized Regression coefficient: $-0.39 \pm 0.11, p<0.001$ ), longer stay in Canada (Standardized Regression coefficient: $-0.23 \pm 0.11, p=0.04$ ), darker skin pigmentation (Standardized Regression coefficient: $0.24 \pm 0.11$, $p=0.027$ ) and low vitamin D intake from food and supplement (Standardized Regression coefficient: $0.46 \pm 0.11, p<0.001$ ) were found to be significantly associated with deficient vitamin D serum status. Both immigrant and refugee children had percentile height (mean \pm SD) lower than the 50th. Height and serum vitamin D were found to be significantly related to the Total Body Bone Mineral Content (Standardized Regression coefficient: $0.13 \pm 0.06, p=0.047$ ), which was low in $41.9 \%$ of migrant children and $35.9 \%$ of refugee children compared to threshold for age, sex, and ethnicity.

Iron status, using the storage protein ferritin as a proxy, was assessed by Sacri et al. [61], who performed a cross-sectional hospital-based study in France on 657 children from 6 months to 6 years with no signs of active inflammation (CRP $<10 \mathrm{mg} / \mathrm{L})$, because it might influence serum ferritin status. $14 \%$ had a migrant mother. Having a migrant (95\% CI: $25.9-34.0)$ or unemployed $(95 \%$ CI: 33.3-44.6) mother was significantly ( $p<0.05$ ) related to low serum ferritin (32 vs $45 \mu \mathrm{g} / \mathrm{L}$ for migrant vs non-migrant mother and $37 \mathrm{vs} 50 \mu \mathrm{g} / \mathrm{L}$ for unemployed vs employed mother). Iron deficiency (ID) prevalence was $2.8 \%$ to $3.2 \%$ depending on serum ferritin threshold of 10 and $12 \mu \mathrm{g} / \mathrm{L}$. Male gender (OR 2.17 (95\% CI: 1-4.70)), mother being a migrant (OR 3.12 (95\% CI: 1.06-9.18)), underprivileged family status (OR 3.83 (95\% CI: 1.39-10.53)) and low maternal education (OR 4.48 (95\% CI: 1.26-15.98)) were related to ID.

However, Saunders et al. [62] found no association between immigrant status and serum ferritin, iron deficiency and iron deficiency anemia in 2614 healthy children aged 12-72 months living in Toronto, $47.6 \%$ of whom had an immigrant family status. The median serum ferritin was $30 \mu \mathrm{g} / \mathrm{L}$ (IQR 19-39); 10.4\% of the children had ID, and 1.9\% had ID anemia. Older age $(p<0.001)$, male sex $(p=0.02)$, no or low cow's milk intake $(p<0.001)$, and shorter breastfeeding duration $(p<0.001)$ were significantly related to higher serum ferritin, while immigrant status was not related to lower levels of serum ferritin. Interestingly, a household income of $£ 15000-£ 29999$ was associated with a $24.1 \%$ increase in child serum ferritin compared to children from families with a higher household income $(p=0.002)$.

Kim et al. [63] analyzed dietary intakes and plasma concentrations of vitamin E, vitamin C, selenium and carotenoid in 29 non-supplemented Latino children aged between 4-8 years migrating to Nebraska, USA. They reported that the mean daily intake of vitamin E was lower than the Estimated Average Requirement (EAR) and Recommended Dietary Allowance (RDA), but none of the subjects consumed less than the RDA for vitamin C ( $25 \mathrm{mg} /$ day), selenium ( $30 \mu \mathrm{g} /$ day), and carotenoid according to the age. Vitamin E plasma concentration was insufficient in 31\% to $69 \%$ of the subjects depending on the criterion used, while mean vitamin $\mathrm{C}$, selenium, and carotenoid concentrations were indicative of adequacy.

\section{Discussion}

This systematic review assessed the association between migration, FI, and major diet-related morbidities in children using data from 28 studies, 26 of whom were observational (cross-sectional, and cohort) studies, while the two others were randomized controlled trials. All but 3 studies were classified as being of good quality (Table 7).

Overweight and obesity were the most investigated problems, but the results were sometimes conflicting, probably because of the different variables (different populations, SES, acculturation, lifestyles ... ) that were taken into account by the single works. Most of the studies highlighted a 
higher prevalence of overweight/obesity in migrant than in non-migrant children [35,37,39,40,42,47-49]. Obesity is linked to difficulties in social adjustment, low self-esteem, depression, and lower academic achievement [64,65], causing a significant reduction in the children's quality of life [66]). Moreover, overweight children are at a greater risk for remaining overweight in adulthood and for the development of chronic illnesses, such as diabetes and cardiovascular diseases [67], which increases premature mortality [68]. Indeed, high cholesterol levels, high triglycerides, and high blood pressure have been observed frequently in migrant children [22,51].

The increased risk of being overweight in children with a migration background is affected by multiple factors, such as maternal obesity [35,40], given that women commonly assume the primary responsibility for the care and feeding of children using familiar lifestyle behaviors [69], lower economic income [50,54,55], and male gender [40]. Indeed, boys, even if generally more active than girls, have been reported to consume fewer vegetables and more sweet drinks compared to girls of the same age and to watch television more hours per day [70], thus unbalancing the ratio between caloric intake and consumption. A lower level of acculturation has been found to be associated with a higher BMI $[42,45,50]$, mainly if both parents were of low educational level [44]. This could be explained, according to other studies, by the fact that unhealthy eating behaviors and sedentary habits are more often observed in children from low educational level families [71,72]. Prevalence of overweight/obesity has been found to be lower in foreign-born children and in children living in the host country for less than 1 year [46,55]. This could be attributed to immigrant self-selection, meaning that the ones who migrate are the healthiest subjects who are able to endure a long and physically demanding journey and who, at the arrival in the host country, are in reasonably good health (the so-called "healthy immigrant effect") [73]. Interestingly, a convergence of migrant health levels to native health ones within approximately 10-20 years has been reported. [73] Moreover, we should consider that BMI is frequently low at the arrival, mostly because in the countries of origin there is not food over-consumption and, in particular, low consumption of fatty, sugary and processed food, that is instead high in the destination countries (although it is true that obesity is increasing worldwide and it is high in some middle-income countries such as Mexico) [74]. The increase in the prevalence of overweight and obesity over time in the host country among migrant children might be due to acquired environmental risk factors (i.e., western-diet, availability of low-cost high-sugar and high-caloric snacks and a sedentary lifestyle) combined with those from the country of origin (i.e., restricting physical activity [49,51], and cultural preferences for large body size as a proxy for wealth, health, or beauty) $[75,76]$. Furthermore, parent's early life deprivation in the countries of origin, where FI and under-nutrition could be significant and dangerous factors to impaired childhood health, has been reported to be associated with parenting behaviors that promote weight gain [77], due to their different perception of what a healthy child weight is [53].

FI appeared to be discordantly correlated to overweight. It could be hypothesized that a higher BMI is easily associated with FI $[41,43]$, given the availability of low-cost energy-dense foods; members of food insecure families report overeating, when possible, foods they like and even dislike to compensate for periods of deficiency [78]. This link could also be explained by the limited knowledge, time and resources that low food-secure subjects experience to engage in healthful eating and physical exercise [79]. It is interesting that the FI-obesity link has been reported to be stronger among women than among children: mothers frequently sacrifice their food supply to ensure that children are food secure, and this is supported by the fact that only half of food insecurity families with children are also child food-insecure. [9]. In contrast, some authors noticed that more food secure migrant families have children with higher BMI than the food insecure ones [38]. Families who can afford to purchase high-cost food such as fruits and vegetables, could also afford to buy more food in general and consequently cause a major intake of calories in children [80].

We also found strong evidence of a link between migration and stunting [22,51,56-58]. Stunting may reflect the cumulative effect of chronic malnutrition and not only under-nutrition [56] and it is associated with increased morbidity and mortality from infection, in particular, pneumonia and 
diarrhea [81], and short and long-term functional impairment, including poor cognition and educational performance in childhood, low productivity and low wages in adulthood [82]. Iriart and colleagues [56] showed a higher prevalence of stunting in overweight children living in families with economic difficulties compared to overweight children who do not experience such conditions, suggesting that being overweight often matches with a high-calorie but nutrient-poor diet. A shorter period of stay in the host country, a higher number of people in the family, being foreign-born, a low educational level, and socio-economic difficulties affect the prevalence of stunting [56-58]. As the years of stay increase, migrants incorporate to the new environment and progressively learn new habits, beliefs, and behaviors, $[57,58]$ and this effect is even more valid in children born in the host country from migrant parents [56]. Probably, by being incorporated into the new environment over time, immigrants learn how to get better food and progressively improve their SES.

Concerning oral health, ECC was found to be more frequent in migrant children from low-income families, and with parents who migrate from non-European countries in their 20s or later. If untreated, ECC evolves to a more severe disease, which can lead to malocclusions, abscesses and pain [21] and can negatively impact on quality of life, nutritional status, and growth [83,84]. It has been also reported that dental problems, left untreated, can accelerate diabetes and cardiovascular disease complications and may be also associated with respiratory diseases and adverse pregnancy outcomes [85].

When migrants arrive in the host country, they face a dental health care system that is predominantly private [86] and most of them cannot access this care due to a lack of financial resources and because they are not covered by dental insurance [87]. Indeed, we found that the other factors that strongly correlated with ECC were family without dental insurance and the absence of a family dentist [59].

A lack of a balanced diet is the cause of micronutrient deficiency, most of all vitamin D deficiency and ID. Serum vitamin D concentration has been noted to be low in a high rate of migrants, due to an inadequacy of vitamin D and calcium intake, and this was significantly connected to low Total Body Mineral Content. Other risk factors include being of female gender and having dark skin pigmentation [60]. Women are usually at a greater risk than men due to religious practices stemming from various parts of the word, causing insufficient skin exposure to the sun. Furthermore, dark skin pigmentation reduces the quantity of vitamin $\mathrm{D}$ produced for a given amount of solar ultraviolet radiation, that is typically lower in high-income countries than in the countries of origin of most migrant people; moreover, light exposure in the host countries is often insufficient during wintertime. Inadequate levels of vitamin D prevent proper bone growth, which can lead to rickets in children [15]. Clinical manifestations of rickets include restlessness, irritability, frontal bossing, wide open fontanelles, skeletal signs (enlargement of costochondral junctions at 6-9 months of age), soft osseous border, teething delayed, profuse sweating, muscle flabby, and upper respiratory tract infections [88]. Skeletal consequences include stunting, developmental motor delay, and deformities. In severe cases, the development of hypocalcemia can lead to tetany, seizures, laryngospasm, cardiomyopathy, and even death [89]. In order to prevent serious disease, consumption of milk and alternatives, vitamin D-fortified food, and vitamin D supplements should be recommended, in addition to regular sun exposure which is also associated with less sedentary lifestyles.

Two studies focused primarily on ID. ID is strictly associated with an inappropriate diet [14], and severe and/or prolonged ID can evolve to anemia. Notably, preschool age children are at a higher risk because of their rapid phase of growth, leading to a rapid depletion of the iron stores when the intake is not sufficient. The occurrence of ID anemia is also adversely affected by intestinal parasitosis [90], whose prevalence has been reported to be around $20 \%$ in newly arrived migrants in an Italian study [91]. We found conflicting results that correlate ID to migration. Strong risk factors raised by these studies are low socio-economic status, high cow's milk intake which is frequently used because infant formulas are more expensive, and longer breastfeeding duration. Iron is an indispensable micronutrient in brain metabolism, and its deficiency can cause changes in the neurotransmitter homeostasis, decrease myelin production, decline synaptogenesis, and decline the function of the basal ganglia, which adversely affect the psychomotor development and mental capacity [92]. ID can also 
affect children's emotional and psychological behavior, and this is correlated with the modification in the metabolism of dopamine, GABA, hippocampal function and structure, and myelinization [93]. ID anemia is also implicated in the susceptibility to infections, mainly of the upper respiratory tract, which are more frequent and have longer duration in anemic than in healthy children [94]. Early identification of ID is important for preventing the potentially irreversible effects of long-term low iron stores, such as developmental delay, mostly in the fields of attention, motivation [95], stimulus encoding, and memory [96]. Preventative strategies involving parents' education about the consumption of naturally iron-rich and of iron-fortified foods since breastmilk weaning could be implemented, as well as the routine exclusion of parasitic infections that can impair iron intestinal absorption and contribute to ID anemia [97].

Most of the studies were performed in the USA, and not much is known about dietary-related problems in children migrating to other areas of the world. The link between overweight/obesity and migration to the USA, mainly in the Latino people, was reported by several authors. However, studies about diet-related morbidities and migration to other countries are scarce and diverse, so that no clear association could be found between specific diseases and migration to specific geographical areas other than Northern America. Moreover, the diverse data which were variably considered in the papers (SES, acculturation, various ethnicities, eating habits, lifestyles) made the studies very heterogeneous between each other and this might account for the, sometimes, conflicting results.

Unhealthy eating habits can undermine healthcare systems. Obesity starting from infancy will probably continue throughout adulthood, and lead to chronic diseases such as diabetes, hypertension, and cardiovascular diseases, which have a strong impact on our healthcare systems [98]. There is solid evidence that programs of screening and interventions could be effective to improve the health of migrant children $[99,100]$. Many pediatric scientific medical societies actively recommend universal screening for social determinants of health, including FI, in order to identify people at risk at a social level. Unfortunately, although tools to efficiently assess social determinants exist, they are still lacking comprehensiveness in providing a concise view of health in socially deprived populations [101-105]. Therefore, public health programs to evaluate the increased frequency of micronutrient deficiencies and diseases in these populations need to be promoted.

\section{Conclusions}

The present systematic review identified a clear association between migration and diet-related morbidities in children, the major risks being obesity and stunting due to chronic malnutrition. FI and frequently low levels of acculturation are important social determinants in migrant people, leading to the purchase of low-cost foods with poor nutritional value and the maintenance of bad food habits and incorrect lifestyles. However, most of the studies were performed in the USA, and not much is known about dietary-related problems in children migrating to other areas of the world; more work should be done to provide clear evidence for other potentially important morbidities in this group.

Health policies should take into account these areas of vulnerability, which might be addressed with specific screening and educational programs. Healthy policies play a key role to face childhood obesity and the related disease, but currently, they are still at an early stage and have no perceptible impact on this problem $[106,107]$. Governments need to implement regulations that make a healthy diet accessible and affordable for all, especially for the most disadvantaged populations at major risk of FI; such changes sound necessary to improve chronic disease prevention and control, from the perspective of the economic sustainability of healthcare [108]. Paying for primary prevention, mostly in childhood, often leads to an overall saving: "Pay now or pay (more) later" [109]. In the same way, integrating oral health into primary care could help increase access to preventive care, treatment, and promote overall health, for example implementing dental hygienists into primary care settings and educating primary care clinicians in the impact of oral diseases, trains them in risk assessment, and how to assimilate oral health preventive services into their practice [110]. 
Patient-centered care and engagement have been reported to lead to greater patient satisfaction, improved clinical outcomes, health service efficiency, and improved health-related business metrics [111]. As Geoffrey Rose stated at the end of the past century, "the primary determinants of disease are mainly economic and social, and, therefore, its remedies must also be economic and social. Medicine and politics cannot and should not be kept apart." [112].

Author Contributions: A.D., D.G. and M.L.: contributed to the design of the review; A.D., V.P., F.M. and D.G.: performed the literature search and data extraction; S.S. and D.G.: performed the quality assessment process; A.D., V.P., F.M., S.S., D.G. and M.L.: contributed to interpretation of findings; A.D., V.P., F.M., S.S., D.G. and M.L.: contributed to the writing of the manuscript. All authors have read and agreed to the published version of the manuscript.

Funding: This research received no external funding.

Conflicts of Interest: The authors declare no conflict of interest.

\section{References}

1. United Nations, Deparment of Economic and Social Affairs, Population Division (2019). World Population Prospects 2019. Available online: https://www.un.org/en/development/desa/population/index.asp (accessed on 26 December 2019).

2. Foresight. Migration and Global Environmental Change; Final project report; The Government Office for Science: London, UK, 2011; pp. 43-45.

3. Holben, D.H. Position of the American Dietetic Association: Food insecurity and hunger in the United States. J. Am. Diet. Assoc. 2006, 106, 446-458. [CrossRef]

4. Cook, J.T.; Frank, D.A.; Levenson, S.M.; Neault, N.B.; Heeren, T.C.; Black, M.M.; Berkowitz, C.; Casey, P.H.; Meyers, A.F.; Cutts, D.B.; et al. Child food insecurity increases risks posed by household food insecurity to young children's health. J. Nutr. 2006, 136, 1073-1076. [CrossRef]

5. Rose-Jacobs, R.; Black, M.M.; Casey, P.H.; Cook, J.T.; Cutts, D.B.; Chilton, M.; Heeren, T.; Levenson, S.M.; Meyers, A.F.; Frank, D.A. Household food insecurity: Associations with at-risk infant and toddler development. Pediatrics 2008, 121, 65-72. [CrossRef]

6. Cook, J.T.; Frank, D.A.; Berkowitz, C.; Black, M.M.; Casey, P.H.; Cutts, D.B.; Meyers, A.F.; Zaldivar, N.; Skalicky, A.; Levenson, S.M.; et al. Food insecurity is associated with adverse health outcomes among human infants and toddlers. J. Nutr. 2004, 134, 1432-1438. [CrossRef]

7. Whitaker, R.C.; Phillips, S.M.; Orzol, S.M. Food insecurity and the risks of depression and anxiety in mothers and behavior problems in their preschool-aged children. Pediatrics 2006, 118, e859-868. [CrossRef]

8. Jyoti, D.F.; Frongillo, E.A.; Jones, S.J. Food insecurity affects school children's academic performance, weight gain, and social skills. J. Nutr. 2005, 135, 2831-2839. [CrossRef]

9. Nord, M.; Andrews, M.S.; Carlson, S. Household food security in the United States, 2008; Dept. of Agriculture, Economic Research Service: Washington, DC, USA, 2008.

10. Capps, R. Hardship among Children of Immigrants: Finding from the National Survey of America's Families; The Urban Institute: Washington, DC, USA, 2001; pp. 1-8.

11. Hernandez, D.J.; College, H.; Napierala, J.S. Children in Immigrant Families: Essential to America's Future. The Foundation for Child Development: New York, NY, USA, 2012; Available online: https://www.fcd-us. org/children-in-immigrant-families-essential-to-americas-future/ (accessed on 26 December 2019).

12. Pettoello-Mantovani, M.; Ehrich, J.; Sacco, M.; Ferrara, P.; Giardino, I.; Pop, T.L. Food in security and children's rights to adequate nutrition in Europe. J. Pediatr. 2018, 198, 329-330. [CrossRef]

13. Audrey, L.; Pereira, L.; Handa, S.; Holmqvist, G. Prevalence and correlates of food insecurity among children across the globe. Available online: https://www.unicef-irc.org/publications/pdf/IWP_2017_09.pdf (accessed on 22 January 2020).

14. Verga, M.E.; Widmeier-Pasche, V.; Beck-Popovic, M.; Pauchard, J.Y.; Gehri, M. Iron deficiency in infancy: Is an immigrant more at risk? Swiss Med. Wkly. 2014, 144, w14065. [CrossRef]

15. Thacher, T.D.; Pludowki, P.; Shaw, N.J.; Mughal, M.Z.; Munns, C.F.; Högler, W. Nutritional rickets in immigrant and refugee children. Public Health Rev. 2016, 37, 3. [CrossRef] 
16. Carter, R.C.; Jacobson, J.L.; Burden, M.J.; Armony-Sivan, R.; Dodge, N.C.; Angelilli, M.L.; Lozoff, B.; Jacobson, S.W. Iron deficiency anemia and cognitive function in infancy. Pediatrics 2010, 126, e427-434. [CrossRef]

17. Renzaho, A.M.; Swinburn, B.; Burns, C. Maintenance of traditional cultural orientation is associated with lower rates of obesity and sedentary behaviours among African migrant children to developed countries. Int. J. Obes. 2008, 32, 594-600. [CrossRef] [PubMed]

18. Roberts, M.W. Dental health of children: Where we are today and remaining challenges. J. Clin. Pediatr. Dent. 2008, 32, 231-234. [CrossRef] [PubMed]

19. Patrick, D.L.; Lee, R.S.; Nucci, M.; Grembowski, D.; Jolles, C.Z.; Milgrom, P. Reducing oral health disparities: A focus on social and cultural determinants. BMC Oral Health 2006, 6, 1-17. [CrossRef]

20. American Academy of Pediatric Dentistry. Reference manual 2004-2005. Pediatr. Dent. 2004, 26, 1-203.

21. Ripa, L.W. Nursing caries: A comprehensive review. Pediatr. Dent. 1988, 10, 262-282.

22. Lane, G.; Farag, M.; White, J.; Nisbet, C.; Vatanparast, H. Chronic health disparities among refugee and immigrant children in Canada. Appl. Physiol. Nutr. Metab. 2018, 43, 1043-1058. [CrossRef]

23. Beiser, M.; Hou, F.; Hyman, I.; Tousignant, M. Poverty, family process, and the mental health of immigrant children in Canada. Am. J. Publ. Health. 2002, 92, 220-227. [CrossRef]

24. Beiser, M. Longitudinal research to promote effective refugee resettlement. Transcult. Psychiatry 2006, 43, 56-71. [CrossRef]

25. Rousseau, C.; Drapeau, A. Are refugee children an at-risk group? A longitudinal study of Cambodian adolescents. J. Refugee Stud. 2003, 16, 67-81. [CrossRef]

26. Moher, D.; Liberati, A.; Tetzlaff, J.; Altman, D.G.; PRISMA Group. Preferred reporting items for systematic reviews and meta-analyses: The PRISMA statement. PLoS Med. 2009, 6, e1000097. [CrossRef]

27. Effective Public Health Practice Project. Quality assessment tool for quantitative studies. Available online: https://merst.ca/wp-content/uploads/2018/02/quality-assessment-tool_2010.pdf (accessed on 19 December 2019).

28. World Health Organization. Consolidated guidelines on the use of antiretroviral drugs for treating and preventing HIV infection. Definition of key terms. Available online: https://www.who.int/hiv/pub/guidelines/ arv2013/intro/keyterms/en/ (accessed on 19 December 2019).

29. OMICS International. Nutritional status research articles. Available online: https://www.omicsonline.org/ nutrition-disorder-and-therapy/nutritional-status-research-articles.php (accessed on 19 December 2019).

30. Miller, M.A.; Zachary, J.F. Chapter 1 Mechanisms and Morphology of Cellular Injury, Adaptation, and Death. In Pathologic Basis of Veterinary Disease, 6th ed.; Zachary, J.F., Ed.; Mosby Elsevier: St. Louis, MI, USA, 2017; pp. 2-43.e19.

31. Food and Agriculture Organization of the United Nations. Food Security Information for Action Practical Guides. An Introduction to the Basic Concepts of Food Security. FAO 2008. Available online: http: //www.fao.org/3/a1936e/a1936e00.pdf (accessed on 19 December 2019).

32. Winkleby, M.A.; Jatulis, D.E.; Frank, E.; Fortmann, S.P. Socioeconomic status and health: How education, income, and occupation contribute to risk factors for cardiovascular disease. Am. J. Public Health 1992, 82, 816-820. [CrossRef]

33. International Organization for Migration. Key migration terms. Available online: https://www.iom.int/keymigration-terms (accessed on 23 December 2019).

34. Besharov, D.J.; Lopez, M.H. Adjusting to a World in Motion: Trends in Global Migration and Migration Policy, 1st ed.; Oxford University Press: New York, NY, USA, 2016; ISBN 978-019-021-139-4.

35. Rosas, L.G.; Guendelman, S.; Harley, K.; Fernald, L.C.; Neufeld, L.; Mejia, F.; Eskenazi, B. Factors associated with overweight and obesity among children of Mexican descent: Results of a binational study. J. Immigr. Minor. Health 2011, 13, 169-180. [CrossRef]

36. Kaiser, L.L.; Aguilera, A.L.; Horowitz, M.; Lamp, C.; Johns, M.; Gomez-Camacho, R.; Ontai, L.; de la Torre, A. Correlates of food patterns in young Latino children at high risk of obesity. Public Health Nutr. 2015, 18, 3042-3050. [CrossRef]

37. Kobel, S.; Lämmle, C.; Wartha, O.; Kesztyüs, D.; Wirt, T.; Steinacker, J.M. Effects of a randomised controlled school-based health promotion intervention on obesity related behavioural outcomes of children with migration background. J. Immigr. Minor. Health 2017, 19, 254-262. [CrossRef] 
38. Kobel, S.; Wirt, T.; Schreiber, A.; Kesztyüs, D.; Kettner, S.; Erkelenz, N.; Wartha, O.; Steinacker, J.M. Intervention effects of a school-based health promotion programme on obesity related behavioural outcomes. J. Obes. 2014, 2014, 1-8. [CrossRef]

39. Early, G.J.; Cheffer, N.D. Motivational interviewing and home visits to improve health behaviors and reduce childhood obesity: A pilot study. Hisp. Health Care Int. 2019, 17, 103-110. [CrossRef]

40. Geremia, R.; Cimadon, H.M.; de Souza, W.B.; Pellanda, L.C. Childhood overweight and obesity in a region of Italian immigration in Southern Brazil: A cross-sectional study. Ital. J. Pediatr 2015, 41, 28. [CrossRef]

41. Distel, L.M.L.; Egbert, A.H.; Bohnert, A.M.; Santiago, C.D. chronic stress and food insecurity: examining key environmental family factors related to body mass index among low-income mexican-origin youth. Fam. Community Health 2019, 42, 213-220. [CrossRef]

42. Buscemi, J.; Beech, B.M.; Relyea, G. Predictors of obesity in Latino children: Acculturation as a moderator of the relationship between food insecurity and body mass index percentile. J. Immigr Minor. Health 2011, 13, 149-154. [CrossRef]

43. Kilanowski, J.F. Patterns and correlates of nutrition among migrant farm-worker children. West. J. Nurs Res. 2012, 34, 396-416. [CrossRef]

44. Ebenegger, V.; Marques-Vidal, P.M.; Nydegger, A.; Laimbacher, J.; Niederer, I.; Bürgi, F.; Giusti, V.; Bodenmann, P.; Kriemler, S.; Puder, J.J. Independent contribution of parental migrant status and educational level to adiposity and eating habits in preschool children. Eur. J. Clin. Nutr. 2011, 65, 210-218. [CrossRef]

45. Huang, X.; Chen, W.; Lin, Y.; Zhang, Q.; Ling, L. Association between acculturation and body weight status among migrant children in Guangzhou, China: A cross-sectional study. BMJ Open 2018, 8, e018768. [CrossRef]

46. Tsujimoto, T.; Kajio, H.; Sugiyama, T. Obesity, diabetes, and length of time in the United States: Analysis of National Health and Nutrition Examination Survey 1999 to 2012. Medicine (Baltimore) 2016, 95, e4578. [CrossRef]

47. Iguacel, I.; Fernández-Alvira, J.M.; Ahrens, W.; Bammann, K.; Gwozdz, W.; Lissner, L.; Michels, N.; Reisch, L.; Russo, P.; Szommer, A.; et al. Prospective associations between social vulnerabilities and children's weight status. Results from the IDEFICS study. Int. J. Obes. (Lond) 2018, 42, 1691-1703. [CrossRef]

48. Labree, W.; van de Mheen, D.; Rutten, F.; Rodenburg, G.; Koopmans, G.; Foets, M. Differences in Overweight and Obesity among Children from Migrant and Native Origin: The Role of Physical Activity, Dietary Intake, and Sleep Duration. PLoS ONE 2015, 10, e0123672. [CrossRef]

49. Besharat Pour, M.; Bergström, A.; Bottai, M.; Kull, I.; Wickman, M.; Håkansson, N.; Wolk, A.; Moradi, T. Effect of parental migration background on childhood nutrition, physical activity, and body mass index. J. Obes. 2014, 406529. [CrossRef]

50. Chomitz, V.R.; Brown, A.; Lee, V.; Must, A.; Chui, K.K.H. Healthy Living Behaviors AmongChinese-American Preschool-Aged Children: Results of a Parent Survey. J. Immigr Minor. Health 2018, 20, 926-935. [CrossRef]

51. Alasagheirin, M.H.; Clark, M.K. Skeletal growth, body composition, and metabolic risk among North Sudanese immigrant children. Public Health Nurs. 2018, 35, 91-99. [CrossRef]

52. Tovar, A.; Hennessy, E.; Pirie, A.; Must, A.; Gute, D.M.; Hyatt, R.R.; Kamins, C.L.; Hughes, S.O.; Boulos, R.; Sliwa, S.; et al. Feeding styles and child weight status among recent immigrant mother-child dyads. Int. J. Behav. Nutr. Phys. Act. 2012, 9, 62. [CrossRef]

53. Cheah, C.S.; Van Hook, J. Chinese and Korean immigrants' early life deprivation: An important factor for child feeding practices and children's body weight in the United States. Soc. Sci. Med. 2012, 74, 744-752. [CrossRef]

54. Cook, W.K.; Tseng, W.; Tam, C.; John, I.; Lui, C. Ethnic-group socioeconomic status as an indicator of community-level disadvantage: A study of overweight/obesity in Asian American adolescents. Soc. Sci. Med. 2017, 184, 15-22. [CrossRef]

55. Zulfiqar, T.; Strazdins, L.; Dinh, H.; Banwell, C.; D’Este, C. Drivers of Overweight/Obesity in 4-11 Year Old Children of Australians and Immigrants; Evidence from Growing Up in Australia. J. Immigr. Minor. Health 2019, 21, 737-750. [CrossRef]

56. Iriart, C.; Handal, A.J.; Boursaw, B.; Rodrigues, G. Chronic malnutrition among overweight Hispanic children: Understanding health disparities. J. Immigr. Minor. Health 2011, 13, 1069-1075. [CrossRef]

57. Choudhary, N.; Parthasarathy, D. Is migration status a determinant of urban nutrition insecurity? Empirical evidence from Mumbai city, India. J. Biosoc. Sci. 2009, 41, 583-605. [CrossRef] 
58. Lee, S.K.; Nam, S.Y.; Hoffman, D. Changes in nutritional status among displaced North Korean children living in South Korea. Ann. Hum. Biol. 2015, 42, 581-584. [CrossRef]

59. Werneck, R.I.; Lawrence, H.P.; Kulkarni, G.V.; Locker, D. Early childhood caries and access to dental care among children of Portuguese-speaking immigrants in the city of Toronto. J. Can. Dent. Assoc. 2008, 74, 805.

60. Vatanparast, H.; Nisbet, C.; Gushulak, B. Vitamin D insufficiency and bone mineral status in a population of newcomer children in Canada. Nutrients 2013, 5, 1561-1572. [CrossRef]

61. Sacri, A.S.; Hercberg, S.; Gouya, L.; Levy, C.; Bocquet, A.; Blondel, B.; Vincelet, C.; Hebel, P.; Vinatier, I.; de Montalembert, M.; et al. Very low prevalence of iron deficiency among young French children: A national cross-sectional hospital-based survey. Matern. Child. Nutr. 2018, 14, e12460. [CrossRef]

62. Saunders, N.R.; Parkin, P.C.; Birken, C.S.; Maguire, J.L.; Borkhoff, C.M.; TARGet Kids! Collaboration. Iron status of young children from immigrant families. Arch. Dis. Child. 2016, 101, 1130-1136. [CrossRef]

63. Kim, Y.N.; Lora, K.R.; Giraud, D.W.; Driskell, J.A. Nonsupplemented children of Latino immigrants have low vitamin $\mathrm{E}$ intakes and plasma concentrations and normal vitamin $\mathrm{C}$, selenium, and carotenoid intakes and plasma concentrations. J. Am. Diet. Assoc. 2006, 106, 385-391. [CrossRef]

64. Datar, A.; Sturm, R.; Magnabosco, J.L. Childhood overweight and academic performance: National study of kindergartners and first-graders. Obes. Res. 2004, 12, 58-68. [CrossRef]

65. Strauss, R.S.; Pollack, H.A. Social marginalization of overweight children. Arch. Pediatr. Adolesc. Med. 2003, 157, 746-752. [CrossRef]

66. Swallen, K.C.; Reither, E.N.; Haas, S.A.; Meier, A.M. Overweight, obesity, and health-related quality of life among adolescents: The national longitudinal study of adolescent health. Pediatrics 2005, 115, 340-347. [CrossRef]

67. Del Rio-Navarro, B.; Velazquez-Monroy, O.; Sanchez-Castillo, C.; Lara-Esqueda, A.; Berber, A.; Fanghanel, G.; Violante, R.; Tapia-Conyer, R.; James, W.P.; Encuesta Nacional de Salud 2000 Working Group; et al. The high prevalence of overweight and obesity in Mexican children. Obes. Res. 2004, 12, 215-223. [CrossRef]

68. Reilly, J.; Kelly, J. Long-term impact of overweight and obesity in childhood and adolescence on morbidity and premature mortality in adulthood: Systematic review. Int. J. Obes. (Lond) 2011, 35, 891-898. [CrossRef]

69. Caprio, S.; Daniels, S.R.; Drewnowski, A.; Kaufman, F.R.; Palinkas, L.A.; Rosenbloom, A.L.; Schwimmer, J.B. Influence of race, ethnicity, and culture on childhood obesity: Implications for prevention and treatment: A consensus statement of Shaping America's Health and the Obesity Society. Diabetes Care 2008, 31, 2211-2221. [CrossRef]

70. Elinder, L.S.; Heinemans, N.; Zeebari, Z.; Patterson, E. Longitudinal changes in health behaviours and body weight among Swedish school children-associations with age, gender and parental education-the SCIP school cohort. BMC Public Health 2014, 14, 640. [CrossRef]

71. Lasserre, A.M.; Chiolero, A.; Cachat, F.; Paccaud, F.; Bovet, P. Overweight in Swiss children and associations with children's and parents' characteristics. Obesity (Silver Spring) 2007, 15, 2912-2919. [CrossRef]

72. Sausenthaler, S.; Kompauer, I.; Mielck, A.; Borte, M.; Herbarth, O.; Schaaf, B.; von Berg, A.; Heinrich, J. Impact of parental education and income inequality on children's food intake. Public Health Nutr. 2007, 10, 24-33. [CrossRef]

73. Kennedy, S.; Kidd, M.P.; McDonald, J.T.; Biddle, N. The healthy immigrant effect: Patterns and evidence from four countries. J. Int. Migr. Integr. 2015, 16, 317-332. [CrossRef]

74. Murphy, M.; Robertson, W.; Oyebode, O. Obesity in International Migrant Populations. Curr. Obes. Rep. 2017, 6, 314-323. [CrossRef]

75. Baker, E.H.; Rendall, M.S.; Weden, M.M. Epidemiological Paradox or Immigrant Vulnerability? Obesity Among Young Children of Immigrants. Demography 2015, 52, 1295-1320. [CrossRef]

76. Gualdi-Russo, E.; Zaccagni, L.; Manzon, V.S.; Masotti, S.; Rinaldo, N.; Khyatti, M. Obesity and physical activity in children of immigrants. Eur. J. Public Health 2014, 24, 40-46. [CrossRef]

77. Kuyper, E.M.; Smith, D.; Kaiser, L.L. Does food insecurity influence child feeding practices? J. Hunger Environ. Nutr 2009, 4, 147-157. [CrossRef]

78. Franklin, B.; Jones, A.; Love, D.; Puckett, S.; Macklin, J.; White-Means, S. Exploring mediators of food insecurity and obesity: A review of recent literature. J. Community Health 2012, 37, 253-264. [CrossRef]

79. Dhurandhar, E.J. The food-insecurity obesity paradox: A resource scarcity hypothesis. Physiol. Behav. 2016, 162, 88-92. [CrossRef] 
80. Sarlio-Lahteenkorva, S.; Lahelma, E. Food insecurity is associated with past and present economic disadvantage and body mass index. J. Nutr. 2001, 131, 2880-2884. [CrossRef]

81. Kossmann, J.; Nestel, P.M.; Herrera, M.G.; El Amin, A.; Fawzi, W.W. Undernutrition in relation to childhood infections: A prospective study in the Sudan. Eur. J. Clin. Nutr. 2000, 54, 463-472. [CrossRef]

82. de Onis, M.; Branca, F. Childhood stunting: A global perspective. Matern. Child. Nutr. 2016, 12, 12-26. [CrossRef]

83. Schroth, R.J.; Harrison, R.L.; Moffatt, M.E. Oral health of indigenous children and the influence of early childhood caries on childhood health and well-being. Pediatr. Clin. N. Am. 2009, 56, 1481-1499. [CrossRef]

84. Clarke, M.; Locker, D.; Berall, G.; Pencharz, P.; Kenny, D.J.; Judd, P. Malnourishment in a population of young children with severe early childhood caries. Pediatr Dent. 2006, 28, 254-259.

85. Bobetsis, Y.A.; Barros, S.P.; Offenbacher, S. Exploring the relationship between periodontal disease and pregnancy complications. J. Am. Dent. Assoc. 2006, 137, 7S-13S. [CrossRef]

86. Birch, S.; Anderson, R. Financing and delivering oral health care: What can we learn from other countries? J. Can. Dent. Assoc. 2005, 71, 243a-243d.

87. Bhatti, T.; Rana, Z.; Grootendorst, P. Dental insurance, income and the use of dental care in Canada. J. Can. Dent. Assoc. 2007, 73, 57.

88. Charoenngam, N.; Shirvani, A.; Holick, M.F. Vitamin D for skeletal and non-skeletal health: What we should know. J. Clin. Orthop. Trauma 2019, 10, 1082-1093. [CrossRef]

89. Holick, M.F. Resurrection of vitamin D deficiency and rickets. J. Clin. Investig. 2006, 116, $2062-2072$. [CrossRef]

90. Yentür Doni, N.; Yıdız Zeyrek, F.; Simşek, Z.; Zeyrek, D. The effects of intestinal parasites on anemia of children. Turkiye Parazitol Derg 2014, 38, 85-90. [CrossRef]

91. Fontanelli Sulekova, L.; Ceccarelli, G.; Pombi, M.; Esvan, R.; Lopalco, M.; Vita, S.; Mattiucci, S.; Gabrielli, S.; Sanitary Bureau of the Asylum Seekers Center of Castelnuovo di Porto. Occurrence of intestinal parasites among asylum seekers in Italy: A cross-sectional study. Travel Med. Infect. Dis. 2019, 27, 46-52. [CrossRef]

92. Vallée, L. Iron and neurodevelopment. Arch. Pediatr. 2017, 24, 5S18-5S22. [CrossRef]

93. Kim, J.; Wessling-Resnick, M. Iron and mechanisms of emotional behavior. J. Nutr. Biochem. 2014, 25, 1101-1107. [CrossRef]

94. de Silva, A.; Atukorala, S.; Weerasinghe, I.; Ahluwalia, N. Iron supplementation improves iron status and reduces morbidity in children with or without upper respiratory tract infections: A randomized controlled study in Colombo, Sri Lanka. Am. J. Clin. Nutr. 2003, 77, 234-241. [CrossRef]

95. Lozoff, B.; Klein, N.K.; Nelson, E.C.; McClish, D.K.; Manuel, M.; Chacon, M.E. Behavior of infants with iron-deficiency anemia. Child. Dev. 1998, 69, 24-36. [CrossRef]

96. Burden, M.J.; Westerlund, A.J.; Armony-Sivan, R.; Nelson, C.A.; Jacobson, S.W.; Lozoff, B.; Angelilli, M.L.; Jacobson, J.L. An event-related potential study of attention and recognition memory in infants with iron-deficiency anemia. Pediatrics 2007, 120, e336-e345. [CrossRef]

97. Carrilho Galvao, F.; Da Costa Gileno, M.; Olivi Malta, J.; Vicentini, V.; Da Costa Gileno, M.; Anibal, F. Anemia in patients with intestinal parasitic infection. Rev. Ibero-Latinoam Parasitol. 2011, 70, 206-211.

98. D'Innocenzo, S.; Biagi, C.; Lanari, M. Obesity and the Mediterranean Diet: A Review of Evidence of the Role and Sustainability of the Mediterranean Diet. Nutrients 2019, 11, 1306. [CrossRef]

99. Berkowitz, S.A.; Hulberg, A.C.; Hong, C.; Stowell, B.J.; Tirozzi, K.J.; Traore, C.Y.; Atlas, S.J. Addressing basic resource needs to improve primary care quality. BMJ Qual. Saf. 2016, 25, 164-172. [CrossRef]

100. Barrett, M.; Combs, V.; Su, J.G.; Henderson, K.; Tuffli, M.; AIR Louisville Collaborative. AIR Louisville: Addressing asthma with technology, crowdsourcing, cross-sector collaboration, and policy. Health Aff. (Millwood) 2018, 37, 525-534. [CrossRef]

101. Schickedanz, A.; Hamity, C.; Rogers, A.; Sharp, A.L.; Jackson, A. Clinician experiences and attitudes regarding screening for social determinants of health in a large integrated health system. Med. Care 2019, 57, S197-S201. [CrossRef]

102. Vest, J.R.; Menachemi, N.; Grannis, S.J.; Ferrell, J.L.; Kasthurirathne, S.N.; Zhang, Y.; Tong, Y.; Halverson, P.K. Impact of risk stratification on referrals and uptake of wraparound services that address social determinants. Am. J. Prev. Med. 2019, 56, e125-e133. [CrossRef] 
103. Buitron de la Vega, P.; Losi, S.; Sprague Martinez, L.; Bovell-Ammon, A.; Garg, A.; James, T.; Ewen, A.M.; Stack, M.; DeCarvalho, H.; Sandel, M.; et al. Implementing an EHR-based screening and referral system to address social determinants of health in primary care. Med. Care 2019, 57, S133-S139. [CrossRef]

104. Tong, S.T.; Liaw, W.R.; Kashiri, P.L.; Pecsok, J.; Rozman, J.; Bazemore, A.W.; Krist, A.H. Clinician experiences with screening for social needs in primary care. J. Am. Board Fam. Med. 2018, 31, 351-363. [CrossRef]

105. The American Board of Family Medicine. PRIME Registry. Available online: https://primeregistry.org/ (accessed on 19 December 2019).

106. Swinburn, B.A. Obesity prevention: The role of policies, laws and regulations. Aust. New Zealand Health Policy 2008, 5, 12. [CrossRef]

107. Gortmaker, S.L.; Swinburn, B.A.; Levy, D.; Carter, R.; Mabry, P.L.; Finegood, D.T.; Moodie, M.L. Changing the future of obesity: Science, policy, and action. Lancet 2011, 378, 838-847. [CrossRef]

108. Mensah, G.A.; Goodman, R.A.; Zaza, S.; Moulton, A.D.; Kocher, P.L.; Dietz, W.H.; Pechacek, T.F.; Marks, J.S. Law as a tool for preventing chronic diseases: Expanding the range of effective public health strategies. Prev. Chronic. Dis. 2004, 1, A13.

109. Grimes, K.E. Lessons From ACEs: Pay Now or Pay (More) Later. Acad. Pediatr. 2017, 17, S18-19. [CrossRef]

110. Stock, R.; Goldberg, B.W. Health Reform Policy to Practice. Oregon's Path to a Sustainable Health System: A Study in Innovation, 1st ed.; Academic Press: Oxford, UK, 2017; Chapter 7; pp. 101-119. ISBN 012-809-827-9.

111. Greaves, F.; Rozenblum, R. Chapter 18: Social and Consumer Informatics. In Key Advances in Clinical Informatics. Transforming Health Care Through Health Information Technology; Aziz Sheikh; Elsevier Science: Amsterdam, The Netherlands, 2017; pp. 257-278.

112. Rose, G.A. The strategy of preventive medicine; Oxford University Press: Oxford, UK, 1992; p. 12. ISBN 0-19-262125-4.

(C) 2020 by the authors. Licensee MDPI, Basel, Switzerland. This article is an open access article distributed under the terms and conditions of the Creative Commons Attribution (CC BY) license (http://creativecommons.org/licenses/by/4.0/). 